This item was submitted to Loughborough's Research Repository by the author.

Items in Figshare are protected by copyright, with all rights reserved, unless otherwise indicated.

\title{
Model-based design of a plant-wide control strategy for a continuous pharmaceutical plant
}

PLEASE CITE THE PUBLISHED VERSION

http://dx.doi.org/10.1002/aic.14107

\section{PUBLISHER}

Wiley (@ 2013 American Institute of Chemical Engineers)

VERSION

SMUR (Submitted Manuscript Under Review)

\section{LICENCE}

CC BY-NC-ND 4.0

\section{REPOSITORY RECORD}

Lakerveld, Richard, Brahim Benyahia, Richard D. Braatz, and Paul I. Barton. 2014. "Model-based Design of a Plant-wide Control Strategy for a Continuous Pharmaceutical Plant". figshare.

https://hdl.handle.net/2134/15277. 
This item was submitted to Loughborough's Institutional Repository (https://dspace.lboro.ac.uk/) by the author and is made available under the following Creative Commons Licence conditions.

\section{creative
commons}

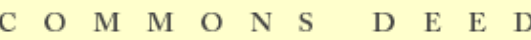

Attribution-NonCommercial-NoDerivs 2.5

You are free:

- to copy, distribute, display, and perform the work

Under the following conditions:

Attribution. You must attribute the work in the manner specified b the author or licensor.

Noncommercial. You may not use this work for commercial purposes.

No Derivative Works. You may not alter, transform, or build upon this work.

- For any reuse or distribution, you must make clear to others the license terms of this work.

- Any of these conditions can be waived if you get permission from the copyright holder.

Your fair use and other rights are in no way affected by the above.

This is a human-readable summary of the Leqal Code (the full license).

\section{Disclaimer 만}

For the full text of this licence, please go to: http://creativecommons.org/licenses/by-nc-nd/2.5/ 


\title{
Model-based design of a plant-wide control strategy for a continuous pharmaceutical plant
}

\author{
Richard Lakerveld, Brahim Benyahia, Richard D. Braatz, and Paul I. Barton* \\ Process Systems Engineering Laboratory, Department of Chemical Engineering, Massachusetts \\ Institute of Technology, Cambridge, Massachusetts, USA \\ E-mail: pib@mit.edu
}

\begin{abstract}
The design of an effective plant-wide control strategy is a key challenge for the development of future continuous pharmaceutical processes. This paper presents a case study for the design of a plant-wide control structure for a system inspired by an end-to-end continuous pharmaceutical pilot plant. A hierarchical decomposition strategy is used to classify control objectives. A plant-wide dynamic model of the process is used to generate parametric sensitivities, which provide a basis for the synthesis of control loops. Simulations for selected disturbances illustrate that the critical quality attributes (CQAs) of the final product can be kept close to specification in the presence of significant and persistent disturbances. Furthermore, it is illustrated how selected CQAs of the final product can be brought simultaneously to a new setpoint while maintaining the remaining CQAs at a constant value during this transition. The latter result shows flexibility to control CQAs independently of each other.
\end{abstract}

\footnotetext{
${ }^{*}$ To whom correspondence should be addressed
} 


\section{Introduction}

The pharmaceutical industry is historically dominated by batch-wise processing. However, the industry is challenged by demands for faster time-to-market, tighter control over product quality, and a smaller ecological foot print. Continuous manufacturing could potentially improve the performance of current pharmaceutical processes as continuous processes are preferred for certain types of chemistry (e.g., see references ${ }^{1-4}$ ), efficient through material and energy recycling, and predictable when combined with real-time monitoring of process and product quality. Cases have been repoerted where significant cost savings can be achieved for continuous manufacturing compared to batch-wise manufacturing. ${ }^{5-13}$ Consequently, there is a strong interest from industry to investigate the potential benefits of new continuous pharmaceutical processes. ${ }^{6,8}$ For example, Roberge et al. ${ }^{7}$ estimated that about $50 \%$ of the reactions they analyzed that were running at Lonza would benefit from continuous processes. However, the transition towards continuous pharmaceutical processing poses also new challenges that need to be addressed. A key challenge is the design of an effective control strategy for continuous pharmaceutical manufacturing, which has to optimize the performance of the plant as a whole instead of isolated unit operations. Specific features of pharmaceutical processes have to be taken into account when approaching this challenge. Pharmaceutical processes are typically characterized by a large variety of unit operations to produce a pharmaceutical product, which can easily result in complicated dynamic behavior once the processes are connected. Furthermore, instead of relying on fixed recipes as is typically done in batch-wise processing, the challenge for control of continuous pharmaceutical processing is to actively maintain the system within a safe region of operation to support regulatory requirements. Disturbances that act on the process can force the system outside the safe region of operation, which has to be prevented by an effective plant-wide control strategy.

Plant-wide control is concerned with the design of a control structure for a complete plant. The questions to be answered concern which variables to measure, manipulate, and control and how to connect them. Buckley ${ }^{14}$ introduces the concept of plant-wide material-balance control by distinguishing between the direction in which the inventories of intermediate storage vessels 
are controlled. Several groups have proposed systematic procedures for the design of plant-wide control structures. Morari et al. ${ }^{15,16}$ approached the systematic development of plant-wide control by introducing a multilayer-multiechelon concept to decompose the control problem into a temporal component by classifying disturbances and a topological component by aggregating interacting groups of processing units with a well-specified operational objective. Temporal classification of disturbances helps to split the control task into an optimizing and a regulating component. Topological classification of the process reduces the complexity of the overall control problem. Larsson and Skogestad ${ }^{17}$ proposed a top-down analysis to select controlled variables followed by a bottomup synthesis of the control loops. Stephanopoulos and $\mathrm{Ng}^{18}$ proposed to utilize a hierarchical view of a plant, such as developed by Douglas ${ }^{19}$ for process synthesis, to facilitate decision making for the design of plant-wide control structures. A hierarchical view decomposes the complexity of the plant-wide control design problem and naturally distributes design tasks over different hierarchical views based on the time scales involved. The separation of time scales has been demonstrated to be beneficial for the design of plant-wide control structures. ${ }^{20}$ The decomposition of a process into different hierarchical views is particularly of interest for continuous pharmaceutical processes as a result of the complexity and various sections for production and purification of intermediates, and formulation of the final drug product.

Control strategies for continuous pharmaceutical processes have to be aligned with Quality-byDesign $(\mathrm{QbD})$ concepts to make the continuous pharmaceutical processes robust to disturbances, uncertainties, and implementation errors. QbD for pharmaceutical processes involves the identification of critical quality attributes (CQAs) and critical process parameters (CPPs). ${ }^{21}$ CQAs are properties of material in the process that need to be controlled within given bounds to ensure a final product quality that is within specification. CPPs are process inputs that have a strong influence on CQAs. Typically, the so-called design space is defined, which is the range of values of all critical process inputs (i.e., input variables and process parameters) for which the process is known to give a product of sufficient quality. ${ }^{22}$ Challenges for the proper use of a design space involve the cost of defining the design space and limited applicability during scale-up. ${ }^{23} \mathrm{~A}$ high 
dimension of the design space often prohibits an exhaustive exploration of the design space, which can result in reduced flexibility for operation, i.e., certain combinations of parameters would give a product of sufficient quality, but are not part of the design space since the favorable outcome has not been proven a priori. Therefore, active control of product properties via feedback and process analytical tools instead of an exhaustive documentation of a design space may well be a more efficient way to develop pharmaceutical processes. ${ }^{23}$ Such active control is likely to be more relevant for continuous pharmaceutical manufacturing since dynamics play a more profound role for integrated continuous processes compared to the dynamics of a chain of individual batch processes. Recently, it was demonstrated, for example, that active feedback control is efficient for a continuous pharmaceutical tablet manufacturing process via roller compaction. ${ }^{24}$

The aim of this paper is to demonstrate the design of a plant-wide control strategy for an end-toend continuous pharmaceutical pilot plant using active feedback control. The system is inspired by a pilot plant that has been built within the Novartis-MIT Center for Continuous Manufacturing and produces a pharmaceutical product from start (synthesis of intermediates) to finish (coating of drug product) in a fully continuous fashion. However, the system does not mimic the real plant exactly. For example, some features such as recycling were not implemented in the real plant. The control strategy aims to exploit hierarchical decomposition to reduce the complexity of the problem and to minimize disturbance propagation between various sections of the plant. A model-based systems approach is of key importance for the development of future continuous pharmaceutical processes. ${ }^{25-27}$ Therefore, a plant-wide dynamic model of the pilot plant ${ }^{28}$ was used to evaluate systematically the influence of CPPs on selected CQAs at each level of the hierarchical decomposition by evaluating parametric sensitivities, which forms the basis for selecting control loops. The resulting control strategy ensures that both plant-wide and local control objectives are effectively addressed with minimum interference due to the hierarchical nature of the control strategy.

The paper is organized as follows. First, the continuous pharmaceutical pilot plant used as a case study in this work is described. The hierarchical decomposition of the process is discussed in the subsequent section including the selection of the control loops at each level of the decomposi- 
tion. The control strategy is implemented in a dynamic model, which is used in the third section to study the performance of the control strategy for selected disturbances and setpoint changes.

\section{Approach}

\section{Process description}

A schematic representation of the pilot plant is in 1 . The process consists of several reactions and separations for synthesis and purification of intermediates and the final active pharmaceutical ingredient (API) followed by a sequence of downstream units that produce a pharmaceutical product in tablet form. The pumps are calibrated volumetric pumps and can be used directly as actuator and as flow indicator. Two reactants $\left(C_{1}, C_{2}\right)$ are mixed at the beginning of the process in a static mixer (M1) with a catalyst and recycled material. The mixture is fed to a tubular reactor (R1) operated at elevated temperature where the chemical reactions

$$
\begin{gathered}
C_{1}+C_{2} \stackrel{\text { Cat }_{1}}{\longrightarrow} C_{3} \\
C_{1} \longrightarrow I_{1} \\
C_{3} \longrightarrow I_{2} \\
\frac{1}{2} C_{1}+\frac{3}{2} C_{2}+\frac{1}{2} C_{3} \longrightarrow I_{3}
\end{gathered}
$$

occur with $C_{3}$ being the desired intermediate compound for production of the final API and compounds $I_{1}, I_{2}$, and $I_{3}$ being undesired byproducts. The reactions 1-4 are relatively slow. An in-line instrument is available at the outlet of the reactor to measure the concentration of both reactants and the intermediate using infrared spectroscopy. Two solvents $\left(S_{1}, S_{2}\right)$ are added in a mixer (M2) at the outlet of the reactor to extract reactant $C_{2}$ and the catalyst from the mixture. The two phases are subsequently separated by a membrane separator (S1). Two flow meters are installed at the outlet of the membrane. The stream with reactant $C_{2}$ and the catalyst is sent to waste and the 
stream with the remaining compounds is introduced into a crystallizer $(\mathrm{Cr} 1)$ operating at low temperature to crystallize the desired intermediate compound $C_{3}$. An anti-solvent $\left(S_{3}\right)$ is added to the first crystallizer $(\mathrm{Cr} 1)$ to lower the solubility of compound $C_{3}$. A second crystallizer $(\mathrm{Cr} 2)$ operated at lower temperature is used to increase yield. Both crystallizers are equipped with a level sensor and a local temperature controller, which maintains the temperature tightly at a fixed value. The crystals are separated from the mother liquor by a combined wash and filtration stage (W1). The permeate of the filtration contains a significant amount of reactant $C_{1}$, which is recycled. A flash evaporator (S2) is used to remove solvent $S_{1}$ from the recycled material. A fraction of the material in the recycle loop is purged (PU1) to prevent excessive buildup of impurities $I_{1-3}$ in the process. A flow meter is installed directly after the purge and the recycled material is fed to the mixer at the beginning of the process (M1).

The purified crystals of intermediate compound $C_{3}$ are partially dissolved in a dilution tank (D1) where solvent $S_{1}$ is added to adjust the concentration of $C_{3}$ for the second reaction. A level indicator and density meter are installed in tank D1. A calibration curve is available to correlate the density of the slurry to the solid fraction of the slurry. An on-line concentration measurement is available to measure the concentration of unconverted reactants, solvent $S_{1}$, and intermediate compound $C_{3}$. A catalyst $\left(\mathrm{Cat}_{2}\right)$ is mixed with the outlet stream of the dilution tank (Stream 26) in a static mixer (M3) and the mixture is fed into a tubular reactor (R2) where the relatively fast chemical reactions

$$
\begin{gathered}
C_{3} \stackrel{\mathrm{Cat}_{2}}{\longrightarrow} C_{4}+I_{4}+G_{1} \\
C_{4} \longrightarrow I_{5}+C_{2} \\
C_{3} \stackrel{\mathrm{Cat}_{2}}{\longrightarrow} I_{4}+I_{6}+G_{1}
\end{gathered}
$$

take place, where $C_{4}$ is the second intermediate compound for production of the final API and compounds $I_{4}, I_{5}$, and $I_{6}$ are undesired byproducts. The outlet stream of the reactor R2 is mixed in 
a static mixer (M4) with compound $C_{5}$ to neutralize the catalyst according to

$$
\mathrm{Cat}_{2}+\mathrm{C}_{5} \longrightarrow \mathrm{C}_{6}
$$

The mixture at the outlet of static mixer M4 consists of two phases, which are separated in a decanter (S3). Two level indicators are installed to measure the level of each phase. The phase created by solvent $S_{2}$ contains mainly reactants $\mathrm{Cat}_{2}, C_{5}$, and byproduct $C_{6}$ and is completely purged (PU3). The phase created by solvent $S_{1}$ contains the intermediate compound $C_{4}$ and byproducts $I_{1-6}$ and is introduced into an adsorption column (S4) to remove traces of solvent $S_{2}$ to lower the solubility of the API in the subsequent crystallization step. An in-line ultraviolet (UV) meter is used at the inlet and the outlet of the adsorption column to measure the concentration of the intermediate compound $C_{4}$. The outlet of the adsorption column is mixed with reactant $C_{7}$ to form the final API in a reactive crystallization $(\mathrm{Cr} 3)$ according to:

$$
C_{4}+\frac{1}{2} C_{7} \longrightarrow A P I
$$

The speed of the API synthesis reaction is very fast compared to the crystallization kinetics. Two crystallizers in series are used with level indicators in both crystallizers. The API crystals are separated from the mother liquor in a combined filtration and washing step (W2) and are subsequently introduced into a dilution tank (D2). A level indicator and density meter are installed in the dilution tank. A calibration curve is available to correlate the density of the slurry to the solid content of the slurry and an on-line concentration measurement is available to measure the concentration of unconverted reactants, solvent $\mathrm{S}_{1}$, and API. The slurry with API crystals is mixed (M5) with an excipient (EX1) to improve flowability of the powder. Drying consists of two steps. First, the bulk of the solvent is removed in a double drum dryer (S5). Subsequently, the solvent content of the powder is brought to a lower value in a screw dryer (S6). The solvent content is measured at the outlet of the screw dryer by a near-infrared (NIR) probe. The dried powder is mixed with a second excipient (EX2) to improve the stability of the final tablet. The excipient is dosed with a 
gravimetric feeder (P24). The mixture is fed to an extruder with a mold (E1) to shape the mixture into a tablet form. A decomposition reaction of the final API occurs within the extruder according to:

$$
A P I \longrightarrow C_{6}+C_{2}
$$

The production of the tablet is finalized by adding a layer of coating material in a continuous coating station (CS). A NIR probe is installed at the end of the process to measure the solvent content, total impurity content, and API dosage of the final tablets. Finally, the production rate of the final product is measured.

[Figure 1 about here.]

\section{Model}

A dynamic model of the pilot plant has been developed to systematically evaluate sensitivities between CPPs and CQAs and to test the performance of various control structures for selected disturbances. The model has been described in detail elsewhere. ${ }^{28}$ A summary of the model including physical properties of the system studied and nominal operating conditions is given in the Supplementary Material for completeness. The values of the parameters are either obtained from experimental data or typical values are assumed. The precise values of model parameters are typically not critical to the performance of a certain control structure. ${ }^{29}$ The core structure of the model is based on mass, energy, and moment balances that aim for at least qualitatively correct behavior, which supports the synthesis and testing of a plant-wide control structure.

\section{Hierarchical decomposition and control objectives}

A combination of a bottom-up and hierarchical top-down approach ${ }^{18,30}$ was used to structure and prioritize the control objectives as shown in 2. First, a bottom-up approach was used to ensure that the underlying process during synthesis of the higher-level control structures is stabilized. Second, 
a top-down hierarchical decomposition was used to structure and prioritize the control objectives that optimize the performance of the control strategy. The importance of selected CPPs at each level of the hierarchical decomposition was evaluated by computing the parametric sensitivities of the CQAs with respect to the CPPs ${ }^{31}$ as given by

$$
S=\frac{\partial y}{\partial P}
$$

where $y$ represents a CQA and $P$ a CPP. Equation (36) can be normalized by introducing the steady-state value for $y$ and nominal value for $P$

$$
\widehat{S}=\frac{\partial y}{\partial P} \frac{P_{n v}}{y_{s t}}
$$

Normalized sensitivities can be beneficial when comparing the influence of several CPPs with different units on a single CQA. The dynamic model including the stabilizing control structure

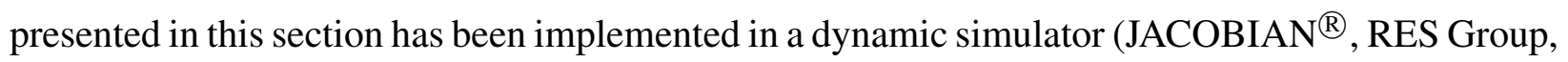
Inc.), which is equipped with efficient methods to compute parametric sensitivities. ${ }^{32,33}$

The decomposition strategy is derived from the approach from Douglas. ${ }^{19}$ At the highest level, only the inputs and outputs of the process are considered. The influences of the CPPs on the CQAs that are visible at the input-output level are evaluated in order to establish constraints that will propagate to lower levels. The process is further decomposed in the second step of the top-down approach where the sections involving synthesis and purification of the first intermediate and API and formulation steps are visualized separately. The influence of the properties of the intermediate streams on the final CQAs are evaluated using parametric sensitivities. Furthermore, strategies are developed to control the identified key properties by utilizing the CPPs available at this level as manipulated variables. The section involving the synthesis and purification of the first intermediate contains a recycle, which is visualized in the third level of the decomposition. The influences of the properties of the streams that become available in this level on the control objectives of this section are analyzed and suitable control strategies are developed. For this study, the control objective is 
to minimize the propagation of disturbances that are acting on the recycle to CQAs at a higher level of the decomposition. Finally, the process is fully represented in the fourth level of the decomposition where remaining degrees of freedom can be used to satisfy local control objectives. The time constants of the relevant processes at higher levels are slow and steady-state information can be used, whereas dynamic modeling has to be utilized at the lowest level to evaluate rejection of faster disturbances. The final control structure of the continuous pharmaceutical pilot plant is obtained by combining each synthesized control layer in a single control structure. A general description of all the steps involved during the application of the design procedure as illustrated in Figure 2 is provided as Supplementary Material. below The application of the proposed strategy to the described pilot plant is discussed in more detail in the subsequent sections.

[Figure 2 about here.]

\section{Stabilizing control structure}

The stabilizing control layer is obtained by identifying all integrating processes and subsequently by synthesizing control loops that stabilize these integrating processes, which involves all of the tanks with an attached pump. The outlet flow rates are used to control the levels of the tanks, as the inlet flow rate is difficult to manipulate for several of the tanks. As a result, the feed flow rates of the process are degrees of freedom for the synthesis of the plant-wide control strategy. 1 gives an overview of all control loops that are part of the stabilizing control layer. All level controllers utilize proportional-control only. The tuning of the control loops will be discussed in the section

involving the synthesis of the most detailed control layer (Level 4). For the first three levels of the decomposition strategy, only steady-state sensitivities are analyzed in order to synthesize the higher-level control loops. The tuning of the level controllers of the stabilizing control layer has little influence on the steady-state behavior of the system.

[Table 1 about here.] 


\section{Level 1: Input/output}

The CQAs that are visible at the input-output level are given in 2 and contain the requirements for the tablets. The CPPs that can influence the CQAs at this level are the composition and flow rates of all of the remaining streams. 3 depicts the computed sensitivities of the CQAs at steady state with respect to the CPPs that are visible at Level 1. The large time constant of the process representation at Level 1 warrants the use of sensitivities at steady state only. The results from 3 can be used to rank the CPPs that have a steady-state effect on the CQAs of the final product, which preferentially are used in subsequent levels to satisfy long-term control objectives. The CPPs that do not have a significant effect on the long-term behavior of the process are preferentially used to satisfy local control objectives at more detailed levels of representation of the process. The solvent content and the API dosage of the final tablets and the production rate are mainly influenced by the flow rate of reactant $C_{1}$, flow rate of solvent $S_{1}$ for the first extraction, flow rate of the second excipient, heat input to the flash evaporator, and purity of the feed stream. The CPPs that have a strong influence on solvent content, API dosage, and production rate also have a strong influence on the total level of impurities of the final tablets. In addition, the purity of the final tablets is significantly influenced by most of the other CPPs that are visible at Level 1 of the decomposition, which likely makes the control of the purity of the final tablets most challenging in subsequent levels. Finally, the analysis confirms that the solvent content of the final tablets is strongly and selectively influenced by the flow rate of solvent $S_{1}$ of Stream 46. Note that the CPPs evaluated in 3 can be used as manipulated variables within their design space to control the CQAs of the final product with exception of the purity of the feeding materials, which is not a practical manipulated variable. It does, however, stress the importance of monitoring changes in the composition of the feed materials, which are quite common for example due to variations in feed material lots or due to variations between suppliers of feed materials. At Level 1 of the decomposition, no direct control loops are established between inputs and outputs as there can potentially be large time delays between controlled and manipulated variables, which will result in sluggish behavior. Instead, control loops involving the important CPPs for long-term behavior are established at the 
next level to ensure sufficiently fast control loops to manipulate the CQAs of the final product as discussed in the next section.

[Table 2 about here.]

[Figure 3 about here.]

\section{Level 2: Intermediates and the formulation of the API}

4 illustrates the process representation at Level 2 of the decomposition. The first block (2A) contains all unit operations that accomplish the synthesis and purification of the first intermediate (compound $C_{3}$ ). The second block $(2 \mathrm{~B})$ is connected to Block $2 \mathrm{~A}$ via Stream 26 and consists of all unit operations that transform the slurry with crystals of intermediate compound $C_{3}$ into a slurry with crystals of the final API (Stream 40). The final block (2C) represents all unit operations involved in the formulation of the API. 4 also contains the control loops that are established at this level, which are the result of the analysis of parametric sensitivities with respect to the CPPs and CQAs that is performed at Level 2. 5 depicts the normalized sensitivities of the CQAs of the final product stream with respect to the properties of the intermediate streams that become visible at Level 2, i.e., the outlet streams of Blocks 2A and 2B (Stream 26 and 40, respectively). The aim is to select those properties that have the strongest influence on the CQAs of the final product stream (plant-wide CQAs), which will become the local CQAs of Level 2. Subsequently, control loops are established around each block to control these CQAs at Level 2, which eventually enables the control of the CQAs of the final product stream at a higher level. 5 shows that there is a clear distinction in the order of magnitude between the sensitivities of the CQAs of the final product with respect to the properties of the intermediate streams that are visible at Level 2 . Therefore, it can be concluded that the CQAs at Level 2 are the weight fraction of solvent $\mathrm{S} 1$, liquid fraction, and flow rate of the slurry with crystals of intermediate compound $C_{3}$ (Stream 26) and equivalently the weight fraction of solvent $\mathrm{S} 1$, liquid fraction, and flow rate of the slurry with crystals of API (Stream 40). 
[Figure 4 about here.]

[Figure 5 about here.]

The three critical properties of Stream 26 must be controlled by utilizing CPPs that are available around Block 2A (4). 6 shows the sensitivities of these local CQAs with respect to the available CPPs. There are several candidate manipulated variables to control the flow rate of Stream 26. The flow rates of Streams 2 and 4, heat input, and purity of Stream 2 all show a sufficiently large and selective sensitivity. The flow rate of Stream 2 was chosen as the manipulated variable to control the flow rate of Stream 26 since purity is not suitable as a manipulated variable and the sensitivity with respect to the flow rate of Stream 2 is the strongest of the remaining CPPs. The liquid fraction of Stream 26 is less sensitive to the CPPs at this level. The selected manipulated variable for the liquid fraction of Stream 26 is the flow rate of Stream 21 as the absolute value of the associated sensitivity is the largest that remains. In addition, the sensitivity of the liquid fraction of Stream 26 with respect to the flow rate of Stream 21 is an order of magnitude larger compared to the sensitivity of the remaining CQA at this level (mass fraction of solvent $S_{1}$ in the liquid phase) with respect to the flow rate of Stream 21. There are three candidate manipulated variables left to control the remaining CQA for Block 2A, which are the flow rates of streams 4 and 7 and the heat input. The use of the heat input is discarded since this CPP has a large influence on all CQAs and it was decided to hold its value constant at Level 2. The flow rate of Stream 7 was chosen as the manipulated variable for the mass fraction of solvent $S_{1}$ in the liquid phase of Stream 26, which yields a slightly better sensitivity compared to using the flow rate of Stream 4. Often several different choices can be made that lead to different control structures. In general, the aim of the approach is not to deliver a single optimized control structure. Different users can come to different control structures that can all be viable choices. Rather, the aim of the approach is to avoid at least those control structures that will clearly result in bad performance due to bad pairings of variables or conflicting local and plant-wide control objectives.

[Figure 6 about here.] 
Note that the qualitative results from 6 are similar to the results in 3 for the CPPs that are evaluated in both graphs. The reason for this similarity is that a variation in a CPP depicted in both figures has to influence the CQAs of Stream 26 first before this variation propagates to the CQAs of the final product. This observation can be generalized to any disturbance that occurs in the first section of the process (i.e., in Block 2A) and the general strategy is to attenuate disturbances such that the influence of the disturbances on local CQAs that have the most effect on the final product is minimized, which prevents upstream disturbances propagating through the whole process. This strategy will be repeated in the next level of the decomposition, which will create another layer of protection against prolonged propagation of disturbances around Block 2A.

The sensitivities of the three CQAs that have to be controlled around Block 2B (4) with respect to the available CPPs are illustrated in 7. The CPPs that are available to control these CQAs are the flow rates of all input streams into Block $2 \mathrm{~B}$ that were also already visible in Level 1 of the decomposition supplemented with the three CQAs of Stream 26 that are input for Block 2B. Using any of these CQAs from Block 2A as a manipulated variable to control the CQAs out of Block 2B would involve changing the setpoints of the control loops that are previously established around Block 2A. 7 shows that several CPPs are suitable, i.e., sufficiently large and selective, to control the flow rate of Stream 40. The flow rate of Stream 26 has been chosen as the manipulated variable to control the flow rate of Stream 40. The use of the liquid fraction or mass fraction of solvent $S_{1}$ in Stream 26 as the manipulated variable to control the flow rate of Stream 40 would be feasible, but the control loops around Block 2A that control these two properties of Stream 26 are expected to saturate easily, which makes the flow rate of Stream 26 a more suitable choice. The flow rate of Stream 37 has been chosen to control the liquid fraction of Stream 40 as the sensitivity of this pair of CQA-CPP is an order of magnitude larger than the sensitivity of the mass fraction of solvent $S_{1}$ in the liquid phase of Stream 40 (remaining CQA) with respect to the flow rate of Stream 37. A similar argument is the basis for selecting the flow rate of Stream 25 as the manipulated variable to control the mass fraction of solvent $S_{1}$ in the liquid phase of Stream 40 as this sensitivity is at least an order of magnitude larger than the sensitivity of the other CQAs with respect to the same CPP. 
[Figure 7 about here.]

Finally, suitable manipulated variables for the CQAs of Block 2C (see 4) have to be identified. The sensitivities of these CQAs with respect to the CPPs that are available around Block $2 \mathrm{C}$ are given in 8. Note that the CQAs of Block 2B are inputs for Block $2 \mathrm{C}$ and the setpoints of the control loops that are established as discussed in the previous section can potentially be used to manipulate the CQAs of Block 2C. The level of total impurities of the final product shows a large sensitivity with respect to the mass-based fraction of solvent $S_{1}$ in the liquid phase of Stream 40, which makes this CPP a suitable choice to establish a control loop around Block 2C to control the level of total impurities in the final product. Note that the sensitivity of the remaining CQAs with respect to this CPP is low. Similarly, the flow rate of Stream 46 is selective to control the solvent content of the final product. The associated control law involving the solvent content and the flow rate of Stream 46 cannot be finalized at this level of the decomposition as suitable actuators are not yet visible, but the control objective that is established at Level 2 will constrain the choices for control loops that can be made at the most detailed level of the decomposition. The two remaining CQAs around Block 2C, i.e., the final API dosage and production rate, show a similar order of magnitude of the sensitivities with respect to the remaining CQAs. Therefore, two CPPs are selected to control those two CQAs and the different priorities between them are used to establish the control laws. The sensitivities of these two remaining CQAs with respect to both the flow rate of Stream 39 and Stream 40 have a reasonably high value. The API dosage of the final tablets is considered to be of a higher priority (see 2) compared to maintaining the production rate. The flow rate of Stream 39 is expected to be a better manipulated variable compared to the flow rate of Stream 40 as the time delay between a change in flow rate of Stream 39 and the time at which a change in one of the CQAs is observed is expected to be shorter and is, therefore, selected as the manipulated variable to control the final API dosage of the tablets around Block 2C. Consequently, the flow rate of Stream 40 is selected as the manipulated variable for controlling the production flow rate, which completes the design of the control structure at Level 2 of the decomposition. The control loops that are established at this level are shown in 4. 
[Figure 8 about here.]

\section{Level 3: Recycle structure}

A control strategy around recycles is developed in Level 3 of the decomposition procedure, which for this application involves expanding Block 2A from Level 2 of the decomposition to reveal the flows that constitute the recycle (see 9). The chosen control strategy aims to reject disturbances within the recycle so as to prevent the propagation of these disturbances to the CQAs of a higher level. The first step is to determine the properties of the streams that are visible at Level 3 and have the largest impact on the CQAs related to Stream 26 as determined at Level 2 of the decomposition. 10 shows the sensitivities of the CQAs of Stream 26 with respect to the weight fractions

of the compounds and flow rates of Streams 9 and 11. Note that the properties of Streams 9 and 11 cannot be treated as parameters in the simulation. In order to generate meaningful sensitivities, parameters are introduced in the model that represent streams of equal composition that can be mixed with Streams 9 and 11. The illustrated sensitivities in 10 represent the sensitivities of the CQAs with respect to an infinitesimal addition of such streams, which mimics perturbations in the composition and flow rate of Streams 9 and 11. Note that Streams 8 and 16 have a similar composition compared to Stream 9 with the exception of the amount of solvent and are excluded from the analysis presented in 10. A clear separation in the order of magnitude of the presented sensitivities can be distinguished. The sensitivity analysis indicates that the fractions of Compounds $C_{1}$ and $C_{3}$ in Streams 9 and 11 and the flow rates of Streams 9 and 11 are expected to have a significant impact on the CQAs of Stream 26. To a lesser extent, the fraction of Compound $C_{2}$ in Stream 11 is expected to have an impact on the CQAs of Stream 26. The fractions of the remaining compounds in Streams 9 and 11 are expected to have a negligible impact on the CQAs of Stream 26. Therefore, the former seven properties of Streams 9 and 11 are designated as the CQAs for Level 3, which must be controlled by a suitable control strategy at Level 3 .

[Figure 9 about here.] 
[Figure 10 about here.]

The second step is to evaluate the sensitivities of the CQAs at Level 3 with respect to the available process parameters at Level 3 of the decomposition, which is presented in 11 . The order of magnitude of the sensitivities with respect to several CPPs is very large, which can make the control of the CQAs at this level impractical. The large sensitivities are a result of the so-called snowball effect, ${ }^{34}$ which is a steady-state phenomenon and implies that a small change in the input parameters of a recycle structure can lead to drastic changes in the state variables of the system as a result of a positive feedback mechanism. In our case, an increase in the amount of solvent that is being recycled will dilute the contents of the reactor, which will lead to a decreased production of intermediate compound $C_{3}$ and consequently more material will be recycled due to dilution of the crystallizers. This mechanism will create a positive feedback in the system since the purge ratio is fixed in the stabilizing control structure that is being analyzed. 12 shows the sensitivities when control of the flow rate of Stream 8 via manipulation of the purge ratio is added to the stabilizing control structure. A stabilizing proportional-integral (PI) controller was used, which will eliminate any steady-state offset. Consequently, the precise tuning of the flow-rate controller does not influence the presented analysis of the sensitivities at steady state. It can be seen that the values of the sensitivities are reduced by an order of magnitude after adding the flow-rate controller to the stabilizing control structure, which allows for synthesis of the control loops at Level 3 of the decomposition. Note that the position of the control valve cannot be used as a manipulated variable anymore and that an additional CPP is available after adding the flow-rate controller, which is the setpoint of the flow-rate controller. The CPPs that are already utilized in control loops at a higher level of the decomposition are the flow rates of Streams 2, 7, and 21. Furthermore, the purities of Streams 2 and 3 are not suitable to be used as manipulated variables and are only included in 12 to illustrate which CQAs are mostly affected by variations in the purity of the feed material. The concentration of compound $C_{2}$ in Stream 9 is controlled by manipulating the flow rate of Stream 3, which has the highest sensitivity compared to the other CQAs. Note that the manipulated variable for this control loop is the setpoint of a ratio control loop, as a change in 
the required flow rate of Stream 3 can be anticipated when the flow rate of Stream 2 is adjusted by the outer control loop that has been developed at Level 2 of the decomposition. The flow rate of Stream 4 provides the highest sensitivity when combined with the concentration of compound $C_{1}$ in the recycle (Stream 11), which defines another control loop. The remaining composition in the recycled stream (compound $C_{3}$ in Stream 11) has been paired to the heat input of the flash evaporator, which has a high sensitivity. Finally, the fraction of compound $C_{3}$ in Stream 9 will be controlled by manipulating the setpoint of the flow-rate controller in the recycle loop. The remaining CQAs are not paired to a manipulated variable as no CPPs with sufficient sensitivity are available to justify additional control loops.

[Figure 11 about here.]

[Figure 12 about here.]

\section{Level 4: Detailed representation}

The remaining degrees of freedom for the synthesis of the plant-wide control structure are fixed in the final step of the hierarchical procedure (see 2), which utilizes the representation of the process at the most detailed level. At this level, local control objectives are satisfied by establishing control loops around individual unit operations. The control loops that are added to the control structure at this level are illustrated in 13 . The flow rate of fresh catalyst that is added to the first reaction (Stream 1) is ratio controlled with the flow rate of reactant $C_{1}$ (Stream 2). The amount of catalyst that is being recycled is negligible as the catalyst dissolves in solvent $S_{2}$ and leaves the process via Stream 20. The other feed streams to the first reactor (Streams 2 and 3) are manipulated at a higher level of the control strategy. The amount of fresh solvent $S_{2}$ that is being added to the mixer (M2) at the outlet of the first reactor $(\mathrm{R} 1)$ is ratio controlled with the flow rate of reactant $C_{2}$ (Stream 3). The reactant $C_{2}$ is added in excess to the first reaction and is the main compound that needs to be extracted by solvent $S_{2}$ at the reactor outlet. The flow rate of solvent $S_{1}$ going into the mixer (Stream 4) is manipulated at a higher level of the control strategy. The crystallizers for separation 
of intermediate compound $C_{3}(\mathrm{Cr} 1, \mathrm{Cr} 2)$ use an anti-solvent to decrease the solubility. An optimal weight fraction of anti-solvent in the crystallizers is maintained by manipulating the flow rate of solvent $S_{3}$ (stream 6) via a measurement of the total flow rate going into the first crystallizer. The flow rate of solvent $S_{1}$ for washing and dilution (Streams 7 and 21, respectively) is manipulated at a higher level of the control strategy.

The flow rate of fresh catalyst $\mathrm{Cat}_{2}$ for the second reaction (Stream 22) is manipulated by the flow rate of Stream 26 and added in excess. The catalyst $\mathrm{Cat}_{2}$ has to be neutralized before Stream 36 can be disposed, which is accomplished by manipulating the flow rate with compound $C_{5}$ (Stream 23) via a ratio control with the flow rate of compound $\mathrm{Cat}_{2}$ going into the second reactor (R2). The first reactive crystallizer $(\mathrm{Cr} 3)$ requires a precise dosing of compound $C_{7}$ with the required stoichiometry in order to crystallize API with a high yield and purity. Therefore, a flow rate measurement and a concentration measurement at the outlet of the adsorption column (S4) are used to determine the amount of compound $C_{7}$ that has to be added via the flow rate of Stream 24 based on the total amount of intermediate compound $C_{4}$ that is being fed into the first crystallizer. The flow rate of solvent $S_{1}$ that is added to the wash and dilution tank (Streams 25 and 37, respectively) is manipulated at a higher level of the control strategy. The first excipient that is being added (Stream 38) aims to improve flowability of the powder and does not have a narrow allowable range for final validation of the product. Therefore, the total flow rate of slurry that enters the dryer is simply used to manipulate the flow rate of Excipient 1 at a fixed ratio. The control objectives for both dryers (S5, S6) are propagated from a higher level of the control strategy. The operating temperature of the screw dryer (S5) can be used as a manipulated variable to adjust the solvent content of the final product. The strategy is to overdesign the residence time of the dryer such that even in the presence of significant inlet disturbances a low solvent content at the outlet of the dryer is obtained. A control loop has been established that will increase the temperature of the dryer only when the solvent content exceeds a certain threshold value, which can occur in the case of large disturbances. Finally, the flow rate of the stream with the second excipient (Stream 39) is manipulated by a control loop at a higher level of the control strategy. 
[Figure 13 about here.]

The design of the control loops at Level 4 of the decomposition completes the synthesis of the plant-wide control structure of the continuous pharmaceutical pilot plant. The complete control structure consists of the control layers that are established at each stage of the design procedure. A drawing of the flowsheet with all control loops is available as Supplementary Material. The control loops have been added to the dynamic model, which is discussed in the next section.

\section{Model-based implementation of the control structure}

\section{Tuning}

The control loops described in previous section are implemented as PI controllers. The tuning of the controllers is based on various criteria. The residence times of all vessels that have a level controller do not need to be controlled tightly in this process, so all of the level controllers only use P control, which results in more gradual changes in the outlet flow rate, compared to PI control, at the expense of a steady-state offset. In addition, a sluggish tuning of the P-controllers or optimal averaging level controllers ${ }^{35}$ can attenuate the propagation of disturbances in flow throughout the process. Fast disturbances entering a unit operation with level control are absorbed more easily and the outer flow-rate control loops established at Level 2 of the decomposition strategy to control the throughput of each section of the plant will mainly observe slower disturbances. The overall strategy is to exploit the buffering capacity of the tanks by having a sloppy proportional control only to reject the short-term disturbances and to utilize a PI controller for the outer flow-rate control loop established at a higher level of the control strategy to reject long-term disturbances. The tuning of the remaining control loops has been accomplished by generating initial guesses for the tuning parameters using the Direct Synthesis Method ${ }^{36}$ by assuming a first-order-plus-time-delay process and manually adjusting those initial parameters in case necessary by evaluating the response of the controllers to typical disturbances via model-based simulations. Significant sources of response delay are reactor R1, adsorption column S4, and screw dryer S6. The PI controllers that become 
saturated easily are implemented in differentiated form to prevent integral windup. An overview of the tuning parameters for all control loops is given in 3 .

[Table 3 about here.]

\section{Performance}

The performance of the plant-wide control structure is tested by simulating various disturbances and observing the dynamic development of the CQAs of the final product. In addition, the ability of the system to sustain changes in setpoints will be investigated. In the first case study, a disturbance is introduced by simulating a gradually decreasing efficiency of pump P2 (Stream 2, which pumps fresh reactant $C_{1}$ to the first mixer). The efficiency of the pump $\eta_{P 2}$ decreases according to

$$
\eta_{P 2}(t)= \begin{cases}1-0.005 t & \text { if } t<40 \\ 0.80 & \text { if } t \geq 40\end{cases}
$$

where $t$ represents time in hours. The dynamic development of the total level of impurities and API in the final product and the production rate are given in 14 for both the closed-loop and open-loop case (i.e., with only a stabilizing control layer). A reduced flow rate of reactant $C_{1}$ will influence both the magnitude and composition of the streams in the system. The illustrated CQAs drop between 10-20\% for the open-loop case. The closed-loop response demonstrates the effectiveness of the control strategy to maintain all CQAs within $2 \%$ from the setpoint in the presence of a significant and persistent input disturbance.

In the second case, a gradual change in reaction kinetics is simulated, which involves a shift in the equilibrium reaction in the first reactor (1) and an increased production of impurity $I_{5}(6)$ in the second reactor as described by

$$
\frac{k_{R 1,1}(t)}{k_{R 1,1}^{0}}= \begin{cases}1-0.005 t & \text { if } t<20 \\ 0.90 & \text { if } t \geq 20\end{cases}
$$




$$
\frac{k_{R 2,2}}{k_{R 2,2}^{0}}= \begin{cases}1+0.010 t & \text { if } t<100 \\ 2.0 & \text { if } t \geq 100\end{cases}
$$

where $k_{R 1,1}$ represents the rate constant of the forward reaction of the equilibrium given by equation (1) and $k_{R 2,2}$ is the rate constant of the reaction give by equation (6). 15 shows the dynamic development of the total level of impurities and API dosage in the final tablets and the production rate for the case with changing reaction kinetics. The total level of impurities increases temporarily to about a maximum of $6 \%$ above the setpoint whereas the same disturbance would produce a permanent increase in the total level of impurities of about $17 \%$ in case only a stabilizing control layer would be present. The production rate shows a smaller deviation and stays within $2 \%$ of the setpoint and the final API dosage remains practically constant throughout the complete run. The disturbance described by equation 15 enters the process in the middle of the process (i.e., in Block 2B from 4). The control loop around Block $2 \mathrm{~B}$ will act to alleviate the effect of the disturbance on the purity of the material going into the dryer (Block 2C) and the control loop around Block 2C will adjust the setpoint of the control loop around Block $2 \mathrm{~B}$ to bring the total level of impurities at steady state back to the desired setpoint.

Finally, the ability of the control strategy to respond to changes in setpoints is demonstrated in 16. The objective is to increase simultaneously the production rate by $30 \%$ and decrease the total level of impurities by $30 \%$. The remaining CQAs of the final product should remain constant during the transition. The results demonstrate that the control strategy is well capable, at least for the demonstrated case, to adjust to new setpoints of selected CQAs of the final product while imposing negligible interfence to the remaining CQAs. The ability to control CQAs independently of each other is of key importance to reduce the complexity for operation and validation of a continuous pharmaceutical plant. It is expected that the illustrated approach can be applied to design a plantwide control structure for other continuous pharmaceutical processes as well. Differences may, however, exist in for example the choices made to decompose the process in different hierarchical viewings, which is dependent on the scope of the design problem. A control system inspired by 
the model-based results presented in this paper has been implemented and tested on a pilot-plant scale and we intend to report those experimental findings in the near future.

[Figure 14 about here.]

[Figure 15 about here.]

[Figure 16 about here.]

\section{Conclusions}

The complexity of continuous pharmaceutical processes motivates the use of systematic procedures for the design of a plant-wide control structure that accounts for the various temporal and structural scales of the process. A combination of a bottom-up and top-down hierarchical approach has been applied to design a plant-wide control structure for a continuous pharmaceutical pilot plant. The bottom-up approach creates a control layer that stabilizes the process and the top-down approach generates several control layers that aim to maintain the critical quality attributes (CQAs) of the final product close to a setpoint. The hierarchical nature of the control layers ensures that the effect of a disturbance is mitigated at the relevant temporal and structural scale of the process. Sensitivity analysis can be used to guide the selection of control loops at various hierarchical levels for the presented case. Simulations of a plant-wide dynamic model including the control structure illustrate that the effect of significant and persistent disturbances can be mitigated at each level of the hierarchical decomposition such that the overall effect of the disturbance on the CQAs of the final product is significantly reduced. Furthermore, a case study illustrates that the system is well capable to respond to a simultaneous change in the setpoint of selected CQAs while the remaining CQAs are not affected during the transition period, which indicates flexibility to control CQAs independently of each other. 


\section{Acknowledgement}

The authors wish to acknowledge Novartis for support. The study presented in this paper is based on a pilot plant that has been constructed within the Novartis-MIT Center for Continuous Manufacturing. The authors wish to acknowledge the members of the team that have developed this pilot plant, in particular: Erin Bell, Stephen Born, Louis Buchbinder, Ellen Cappo, Josh Dittrich, James M.B. Evans, Patrick L. Heider, Devin R. Hersey, Rachael Hogan, Ashley King, Tushar Kulkarni, Aaron Lamoureux, Salvatore Mascia, Sean Ogden, Joel Putnam, Justin Quon, Min Su, Kristen Talbot, Mengying Tao, Chris Testa, Forrest Whitcher, Aaron Wolfe, and Haitao Zhang.

\section{Supplementary Material}

The following material is available online as Supplementary Material:

1. A summary of the process model used including an overview of the physical properties and nominal operating conditions for the system,

2. the numerical values for the sensitivities presented in this paper,

3. a flowsheet that illustrates all control loops,

4. a generic step-by-step description of the followed approach.

\section{References}

(1) Kockmann N, Gottsponer M, Zimmermann B, Roberge DM. Enabling ContinuousFlow Chemistry in Microstructured Devices for Pharmaceutical and Fine-Chemical Production. Chem. Eur. J. 2008;14:7470-7477.

(2) Hartman RL, McMullen JP, Jensen KF. Deciding Whether To Go with the Flow: Evaluating the Merits of Flow Reactors for Synthesis. Angew. Chem. Int. Ed. 2011;50:75027519. 
(3) Wegner J, Ceylan S, Kirschning A. Ten key issues in modern flow chemistry. Chem. Commun. 2011;47:4583-4592.

(4) Wegner J, Ceylan S, Kirschning A. Flow Chemistry - A Key Enabling Technology for (Multistep) Organic Synthesis. Adv. Synth. Catal. 2012;354:17-57.

(5) Schaber SD, Gerogiorgis DI, Ramachandran R, Evans JMB, Barton PI, Trout BL. Economic analysis of integrated continuous and batch pharmaceutical manufacturing: A case study. Ind. Eng. Chem. Res. 2011;50:10083-10092.

(6) Plumb K. Continuous Processing in the Pharmaceutical Industry: Changing the Mind Set. Chem. Eng. Res. Des. 2005;83:730-738.

(7) Roberge DM, Ducry L, Bieler N, Cretton P, Zimmermann B. Microreactor Technology: A Revolution for the Fine Chemical and Pharmaceutical Industries?. Chem. Eng. Technol. 2005;28:318-323.

(8) Roberge DM, Zimmermann B, Rainone F, Gottsponer M, Eyholzer M, Kockmann N. Microreactor technology and continuous processes in the fine chemical and pharmaceutical industry: Is the revolution underway? Org. Process Res. Dev. 2008;12:905910.

(9) Jimenez-Gonzalez C, Poechlauer P, Broxterman QB, Yang BS, Am Ende D, Baird J, Bertsch C, Hannah RE, Dell'Orco P, Noorrnan H, Yee S, Reintjens R, Wells A, Massonneau V, Manley J. Key Green Engineering Research Areas for Sustainable Manufacturing: A Perspective from Pharmaceutical and Fine Chemicals Manufacturers. Org. Process. Res. Dev. 2011;15:900-911.

(10) LaPorte TL, Wang C. Continuous processes for the production of pharmaceutical intermediates and active pharmaceutical ingredients. Curr. Opin. Drug Discovery Dev. 2007;10:738-745. 
(11) Pollet P, Cope ED, Kassner MK, Charney R, Terett SH, Richman KW, Dubay W, Stringer J, Eckertt CA, Liotta CL. Production of (S)-1-Benzyl-3-diazo-2oxopropylcarbamic Acid tert-Butyl Ester, a Diazoketone Pharmaceutical Intermediate, Employing a Small Scale Continuous Reactor. Ind. Eng. Chem. Res. 2009;48:70327036.

(12) Christensen KM, Pedersen MJ, Dam-Johansen K, Holm TL, Skovby T, Kiil S. Design and operation of a filter reactor for continuous production of a selected pharmaceutical intermediate. Chem.Eng.Sci. 2012;26:111-117.

(13) Cervera-Padrell AE, Skovby T, Kiil S, Gani R, Gernaey KV. Active pharmaceutical ingredient (API) production involving continuous processes Ú A process systems engineering (PSE)-assisted design framework. Eur. J. Pharm. Biopharm. 2012;82:437-456.

(14) Buckley PS. Techniques of Process Control. New York: John Wiley \& Sons, 1964.

(15) Morari M, Arkun Y, Stephanopoulos G. Studies in the synthesis of control structures for chemical processes: Part 1. Formulation of the problem. Process decomposition and the classification of the control tasks. Analysis of the optimizing control structures. AIChE J. 1980;26:220-232.

(16) Morari M, Stephanopoulos G. Studies in the synthesis of control structures for chemical processes: Part II. Structural aspects and the synthesis of alternative feasible control schemes. AIChE J. 1980;26:232-246.

(17) Larsson T, Skogestad S. Plantwide control - A review and a new design procedure. Int. J. Model. Ident. Control 2000;21:209-240.

(18) Stephanopoulos G, Ng C. Perspectives on the synthesis of plant-wide control structures. J. Process Control 2000;10:97-111. 
(19) Douglas JM. A hierarchical decision procedure for process synthesis. AIChE J. $1985 ; 31: 353-362$.

(20) Baldea M, Daoutidis P. Control of integrated process networks - A multi-time scale perspective. Comput. Chem. Eng. 2007;31:426-444.

(21) Yu LX. Pharmaceutical quality by design: Product and process development, understanding, and control. Pharm. Res. 2008;25:781-791.

(22) ICH. Guidance for Industry Q8(R2) Pharmaceutical Development. 2009

(23) Lionberger RA, Lee SL, Lee L, Raw A, Yu LX. Quality by Design: Concepts for ANDAs. The AAPS Journal 2008;10:268-276.

(24) Singh R, Ierapetritou M, Ramachandran R. An engineering study on the enhanced control and operation of continuous manufacturing of pharmaceutical tablets via roller compaction. Int. J. Pharm. 2012;438:307-326.

(25) Gernaey KV, Gani R. A model-based systems approach to pharmaceutical productprocess design and analysis. Chem. Eng. Sci. 2010;65:5757-5769.

(26) Gernaey KV, Cervera-Padrell AE, Woodley JM. Development of continuous pharmaceutical production processes supported by process systems engineering methods and tools. Future Med. Chem. 2012;4:1371-1374.

(27) Boukouvala F, Niotis V, Ramachandran R, Muzzio FJ, Ierapetritou MG. An integrated approach for dynamic flowsheet modeling and sensitivity analysis of a continuous tablet manufacturing process. Comput. Chem. Eng. 2012;42:30-47.

(28) Benyahia B, Lakerveld R, Barton PI. A plant-wide dynamic model of a continuous pharmaceutical process. Ind. Eng. Chem. Res. 2012;51:15393-15412.

(29) Skogestad S. Control structure design for complete chemical plants. Comput. Chem. Eng. 2004;28:219-234. 
(30) Ng CS, Stephanopoulos G. Synthesis of control systems for chemical plants. Comput. Chem. Eng. 1996;20:S999-S1004.

(31) Singh R, Gernaey KV, Gani R. Model-based computer-aided framework for design of process monitoring and analysis systems. Comput. Chem. Eng. 2009;33:22-42.

(32) Feehery WF, Tolsma JE, Barton PI. Efficient sensitivity analysis of large-scale differential-algebraic systems. Appl. Numer. Math. 1997;25:41-54.

(33) Galan S, Feehery WF, Barton PI. Parametric sensitivity functions for hybrid discrete/continuous systems. Appl. Numer. Math. 1999;31:17-47.

(34) Luyben WL. Snowball Effects in Reactor/Separator Processes with Recycle. Ind. Eng. Chem. Res. 1994;33:299-305.

(35) McDonald KA, McAvoy TJ, Tits A. Optimal Averaging Level Control. AIChE J. $1986 ; 32: 75-86$.

(36) Seborg DE, Edgar TF, Mellichamp DA. Process Dynamics and Control. New York: John Wiley \& Sons, 1989. 


\section{List of Figures}

1 Process flowsheet . . . . . . . . . . . . . . . . .

2 Schematic representation of the procedure used to develop a plant-wide control strategy for the continuous pharmaceutical pilot plant. The procedure started with a bottom-up approach to stabilize the process followed by a top-down hierarchical approach to develop control strategies at different representations of the process. Level 1 focuses on the input and output streams of the plant, which is decomposed in Level 2 into separate sections for the synthesis and purification of intermediates and solid processing. Recycle structures are revealed during Level 3 of the decomposition. Finally, control strategies at the most detailed level are developed during Level 4 of the approach. The control strategies developed at higher levels constrain the control strategies at lower levels. The hierarchical nature of the approach aims to satisfy both plant-wide and local control objectives with minimum interference. . . . . . . .

3 Steady-state values of the normalized sensitivities of the CQAs with respect to the CPPs at Level 1 . The stream numbers that are used to explain the CPPs refer to 1 . The numerical values of the sensitivities are available as Supplementary Material. . . . . . . . . . . . . .

4 Representation of the continuous pharmaceutical pilot plant at Level 2 of the decomposition strategy. Block $2 \mathrm{~A}$ entails all unit operations related to production of the first intermediate compound. Block $2 \mathrm{~B}$ is dedicated to the synthesis and purification of the final API and Block $2 \mathrm{C}$ involves solids processing. The control loops aim to control the properties of the intermediate streams that are critical to the quality of the final product. . . . . . . . . . . . . . .

5 Steady-state values of the normalized sensitivities of the CQAs with respect to the CPPs at Level 2. The stream numbers that are used to explain the CPPs refer to 1 . The fraction of the various compounds in the table with CPPs refers to the mass-based fraction of the liquid phase. The liquid fraction (CPP \#11 and \#33) is the volume-based liquid fraction of the slurry. The numerical values of the sensitivities are available as Supplementary Material. . . . . . . . . . . . . . . . .

6 Steady-state values of the normalized sensitivities of CPPs of Stream 26 that have the largest impact on the CQAs of the final product with respect to the available manipulated variables for Block $2 \mathrm{~A}$ of the decomposition (see 5). The liquid fraction is the volume-based liquid fraction of the slurry. The numerical values of the sensitivities are available as Supplementary Material. . . . . . . . . . . . . . . . . 
7 Steady-state values of the normalized sensitivities of CPPs of Stream 40 that have the largest impact on the CQAs of the final product with respect to the available manipulated variables for Block $2 \mathrm{~B}$ of the decomposition (see 5). In the legend, "Fraction $S_{1}$ " refers to the massbased fraction of solvent $S_{1}$ in the liquid phase and "Liquid fraction" refers to the volume-based liquid fraction of the slurry. The CPPs with ID 6,7 , and 8 can be manipulated by changing the setpoints of control loops that are established around Block 2A (see 4). The numerical values of the sensitivities are available as Supplementary Material. . . Steady-state values of the normalized sensitivities of the CQAs of the final product with respect to the available manipulated variables for Block $2 \mathrm{C}$ of the decomposition (see 5). In the legend, "Fraction $\mathrm{S}_{1}$ " refers to the mass-based fraction of solvent $S_{1}$ in the liquid phase and "Liquid fraction" refers to the volume-based liquid fraction of the slurry. The CPPs with ID 4, 5, and 6 can be manipulated by changing the setpoints of control loops that are established around Block 2A (see 4). The numerical values of the sensitivities are available as Supplementary Material. . . . . . . . . . . . . . . . . Representation of the continuous pharmaceutical pilot plant at Level 3 of the decomposition strategy. Block 2 A from Level 2 (see 4) has been expanded to visualize the recycle structure. Block $3 \mathrm{~A}$ contains all unit operations related to the first reaction, which produces the intermediate impurity $\mathrm{I}_{1}$. Block $3 \mathrm{~B}$ represents all unit operations that are used to separate the reaction products into a stream that is rich in impurity $\mathrm{I}_{1}$ (Stream 26) and a stream that is rich in reactant $C_{1}$ (Stream 16), which is partially purged (Stream 18) and send to a flash evaporator (Block 3C) to remove solvent $S_{1}$ (Stream 19) before the material returns to the reaction section (Stream 9). The control loops aim to control the CQAs of the streams that constitute the recycle to minimize the propagation of disturbances to the CQAs of Stream 26, which are critical at a higher level of the decomposition. . . . . . . . . . . . . . . . .

10 Steady-state values of the normalized sensitivities of the CQAs of Level 2 with respect to the CPPs at Level 3. The stream numbers refer to 1 . The fraction of the various compounds in the table with CPPs represents the mass-based fraction of a compound in the liquid phase. The liquid fractions are volume-based liquid fraction of a slurry. The numerical values of the sensitivities are available as Supplementary Material. . . . . . . . . . . . . . . . . . .

11 Steady-state values of the normalized sensitivities of CPPs of Streams 9 and 11 that have the largest impact on the CQAs of Stream 26 (see 10). The liquid fraction is the volume-based liquid fraction of the slurry. The numerical values of the sensitivities are available as Supplementary Material. . . . . . . . . . . . . . . . . 
12 Steady-state values of the normalized sensitivities of CPPs of Streams 9 and 11 that have the largest impact on the CQAs of Stream 26 (see 10) with a controlled flow rate in the recycle. The liquid fraction is the volume-based liquid fraction of the slurry. The numerical values of the sensitivities are available as Supplementary Material. . . . . . . . . .

13 Representation of the continuous pharmaceutical pilot plant at the most detailed level of the decomposition strategy. The control structure contains all stabilizing control loops and the control loops that are synthesized at Level 4 of the decomposition to satisfy local control objectives. 44

14 Dynamic development of three CQAs of the final product stream for a disturbance in the flow rate of fresh reactant $C_{1}$ (Case I). The black lines illustrate the closed-loop response and the gray lines illustrate the response with a stabilizing control layer only. The solid lines represent the dynamic development of the total level of impurities, the dotted lines represent the dynamic development of the API dosage, and the dashed lines represent the dynamic development of the production rate. Dynamic development of three CQAs of the final product stream for a gradual change in the kinetics of the reactions in reactor 1 and 2 (Case II). The black lines illustrate the closed-loop response and the gray lines illustrate the response with a stabilizing control layer only. The solid lines represent the dynamic development of the total level of impurities, the dotted lines represent the dynamic development of the API dosage, and the dashed lines represent the dynamic development of the production rate. . . . . . . . . . . . . . .

16 Dynamic development of four CQAs of the final product stream as a result of a step-change in the setpoints of the production rate and total level of impurities at $t=0(+30 \%$ and $-30 \%$ respectively). The solid black line represents the dynamic development of the total level of impurities, the dotted black line (circles) represents the dynamic development of the API dosage, the dotted gray line (squares) represents the dynamic development of the solvent content, and the dashed black line represents the dynamic development of the production rate. . . . . . 


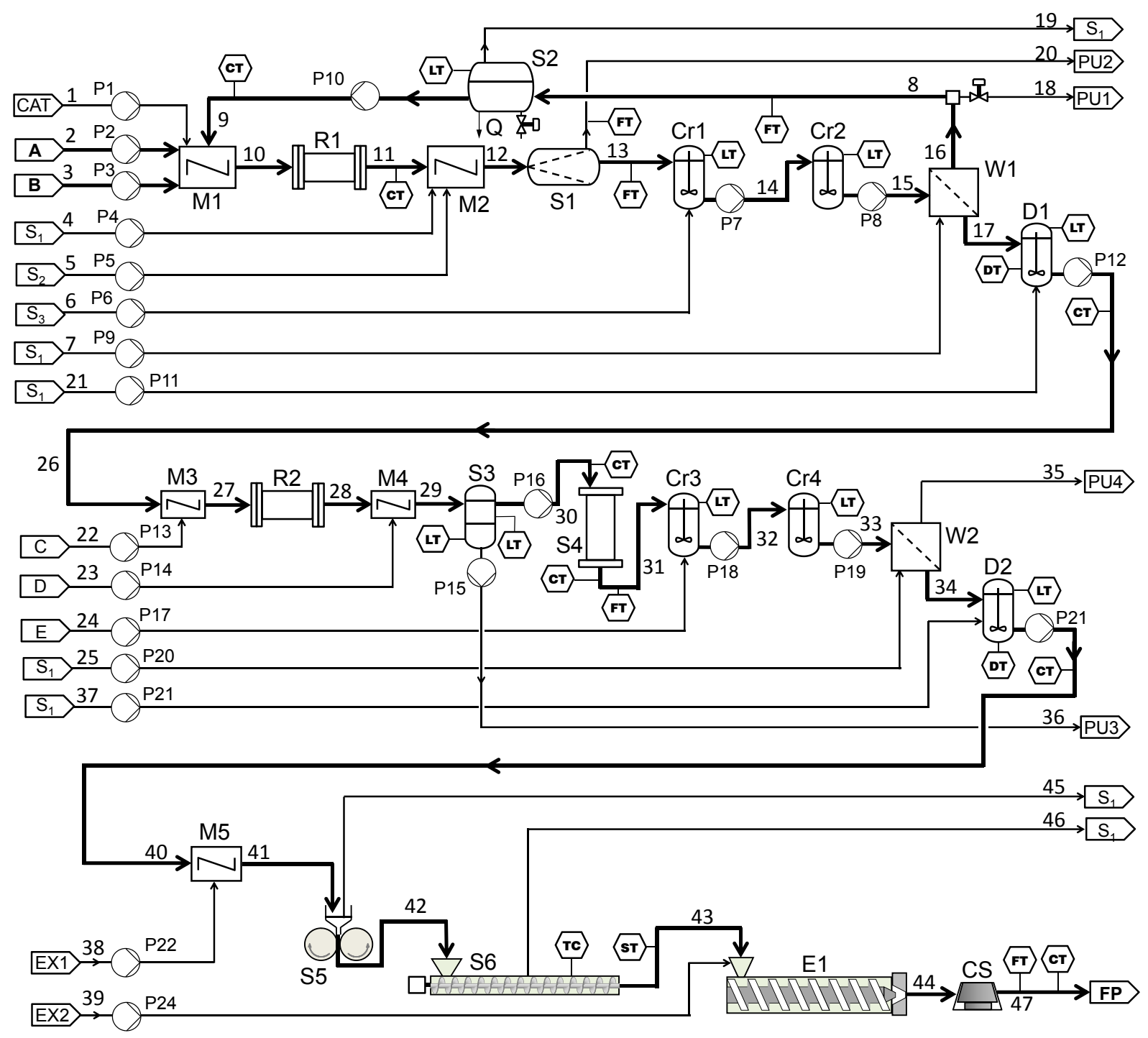

Figure 1: Process flowsheet 


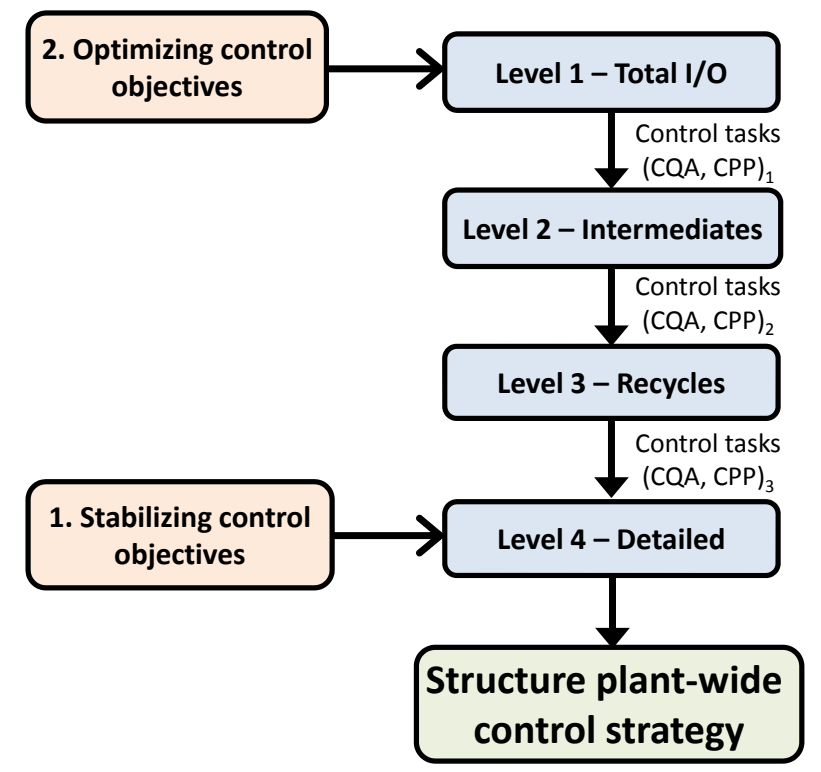

Figure 2: Schematic representation of the procedure used to develop a plant-wide control strategy for the continuous pharmaceutical pilot plant. The procedure started with a bottom-up approach to stabilize the process followed by a top-down hierarchical approach to develop control strategies at different representations of the process. Level 1 focuses on the input and output streams of the plant, which is decomposed in Level 2 into separate sections for the synthesis and purification of intermediates and the formulation of the API. Recycle structures are revealed during Level 3 of the decomposition. Finally, control strategies at the most detailed level are developed during Level 4 of the approach. The control strategies developed at higher levels constrain the control strategies at lower levels. The hierarchical nature of the approach aims to satisfy both plant-wide and local control objectives with minimum interference. 


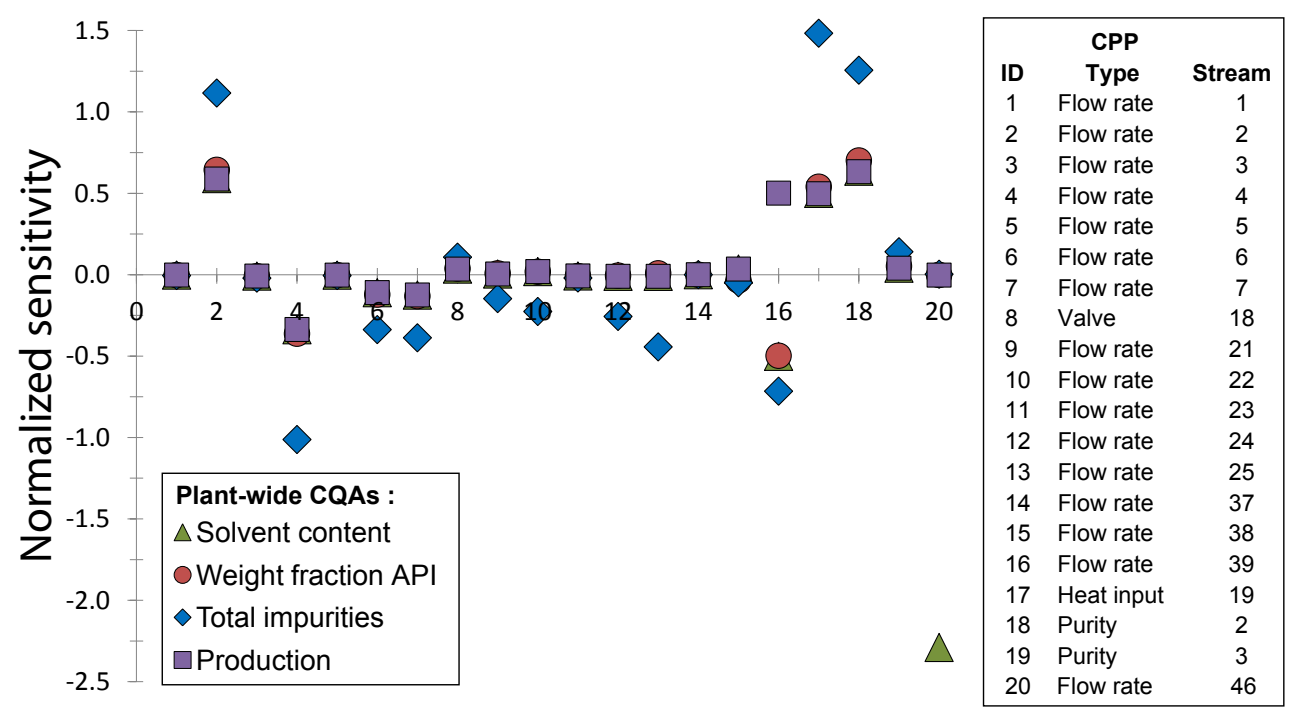

Figure 3: Steady-state values of the normalized sensitivities of the CQAs with respect to the CPPs at Level 1. The stream numbers that are used to explain the CPPs refer to 1 . The numerical values of the sensitivities are available as Supplementary Material. 


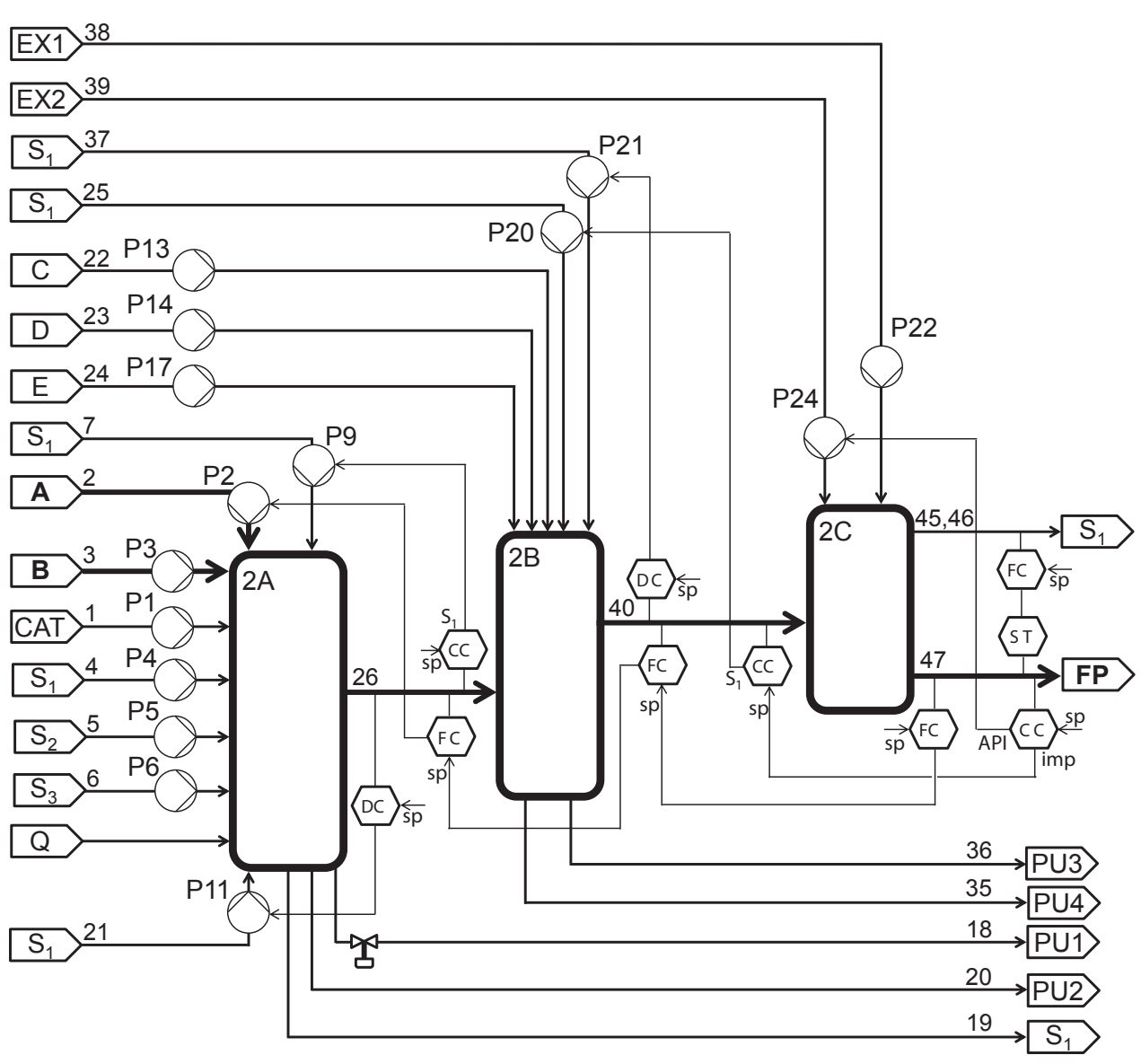

Figure 4: Representation of the continuous pharmaceutical pilot plant at Level 2 of the decomposition strategy. Block 2A entails all unit operations related to production of the first intermediate compound. Block $2 \mathrm{~B}$ is dedicated to the synthesis and purification of the final API and Block $2 \mathrm{C}$ involves the formulation of the API. The control loops aim to control the properties of the intermediate streams that are critical to the quality of the final product. 


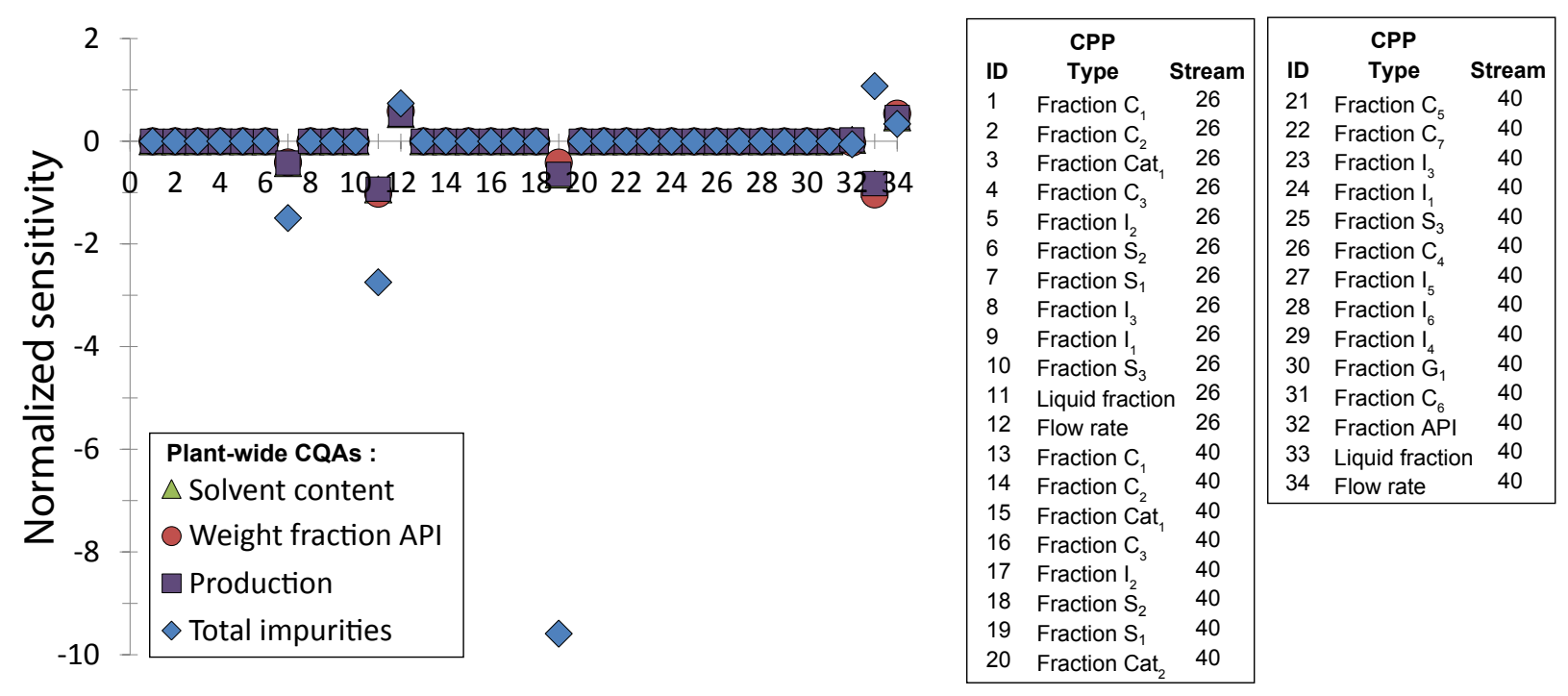

Figure 5: Steady-state values of the normalized sensitivities of the CQAs with respect to the CPPs at Level 2. The stream numbers that are used to explain the CPPs refer to 1 . The fraction of the various compounds in the table with CPPs refers to the mass-based fraction of the liquid phase. The liquid fraction (CPP \#11 and \#33) is the volume-based liquid fraction of the slurry. The numerical values of the sensitivities are available as Supplementary Material. 


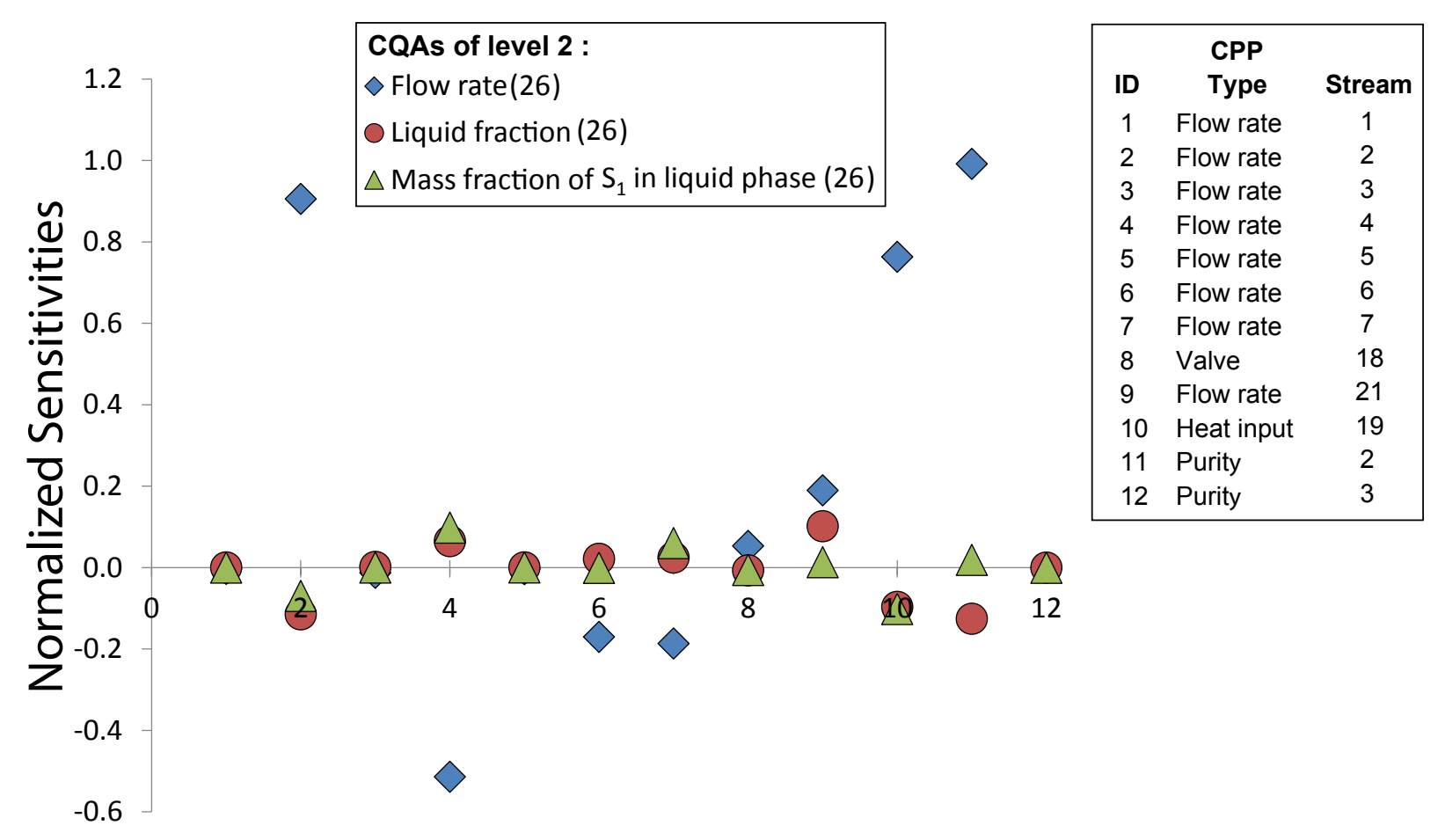

Figure 6: Steady-state values of the normalized sensitivities of CPPs of Stream 26 that have the largest impact on the CQAs of the final product with respect to the available manipulated variables for Block 2A of the decomposition (see 5). The liquid fraction is the volume-based liquid fraction of the slurry. The numerical values of the sensitivities are available as Supplementary Material. 


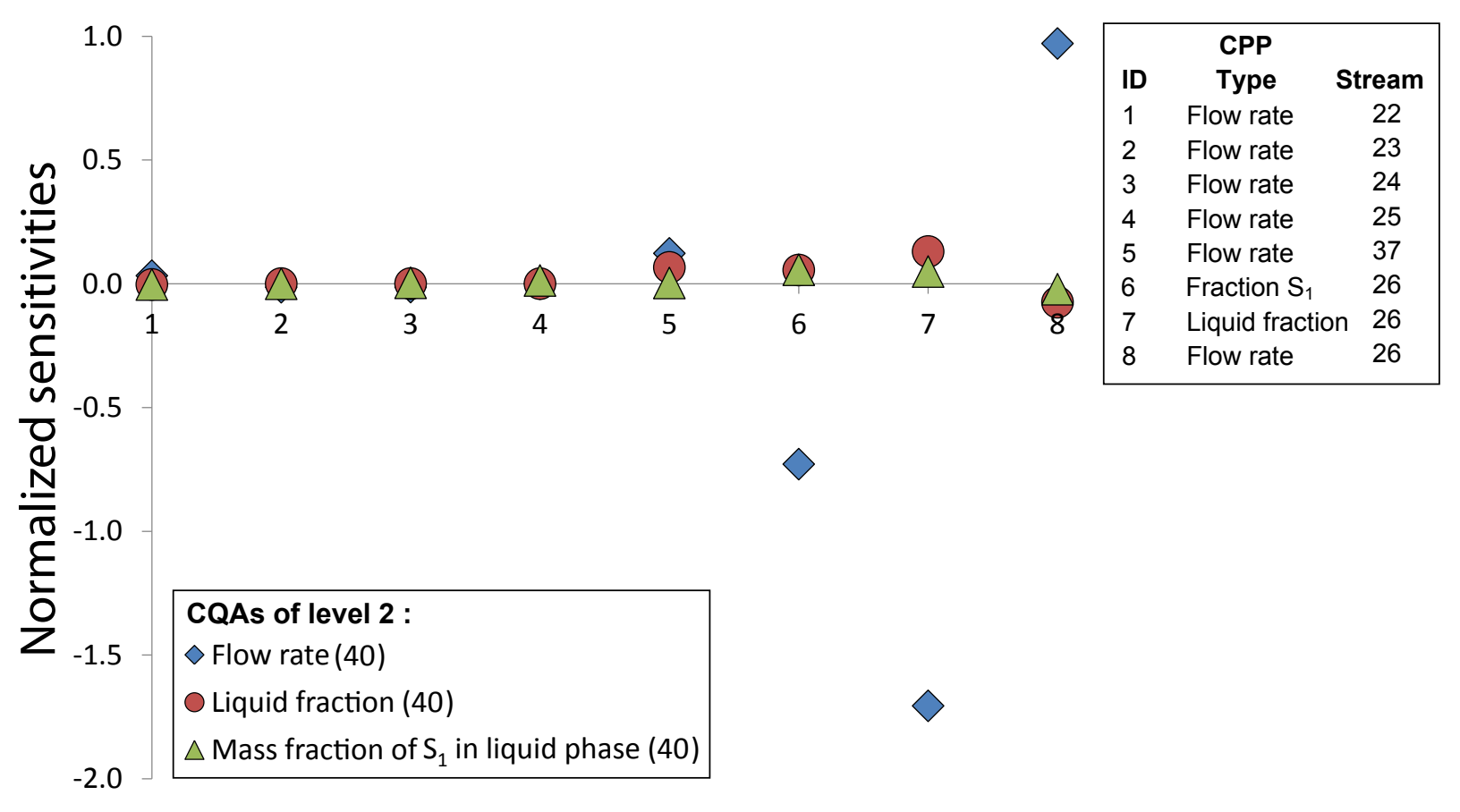

Figure 7: Steady-state values of the normalized sensitivities of CPPs of Stream 40 that have the largest impact on the CQAs of the final product with respect to the available manipulated variables for Block 2B of the decomposition (see 5). In the legend, "Fraction $S_{1}$ " refers to the mass-based fraction of solvent $S_{1}$ in the liquid phase and "Liquid fraction" refers to the volume-based liquid fraction of the slurry. The CPPs with ID 6, 7, and 8 can be manipulated by changing the setpoints of control loops that are established around Block 2A (see 4). The numerical values of the sensitivities are available as Supplementary Material. 


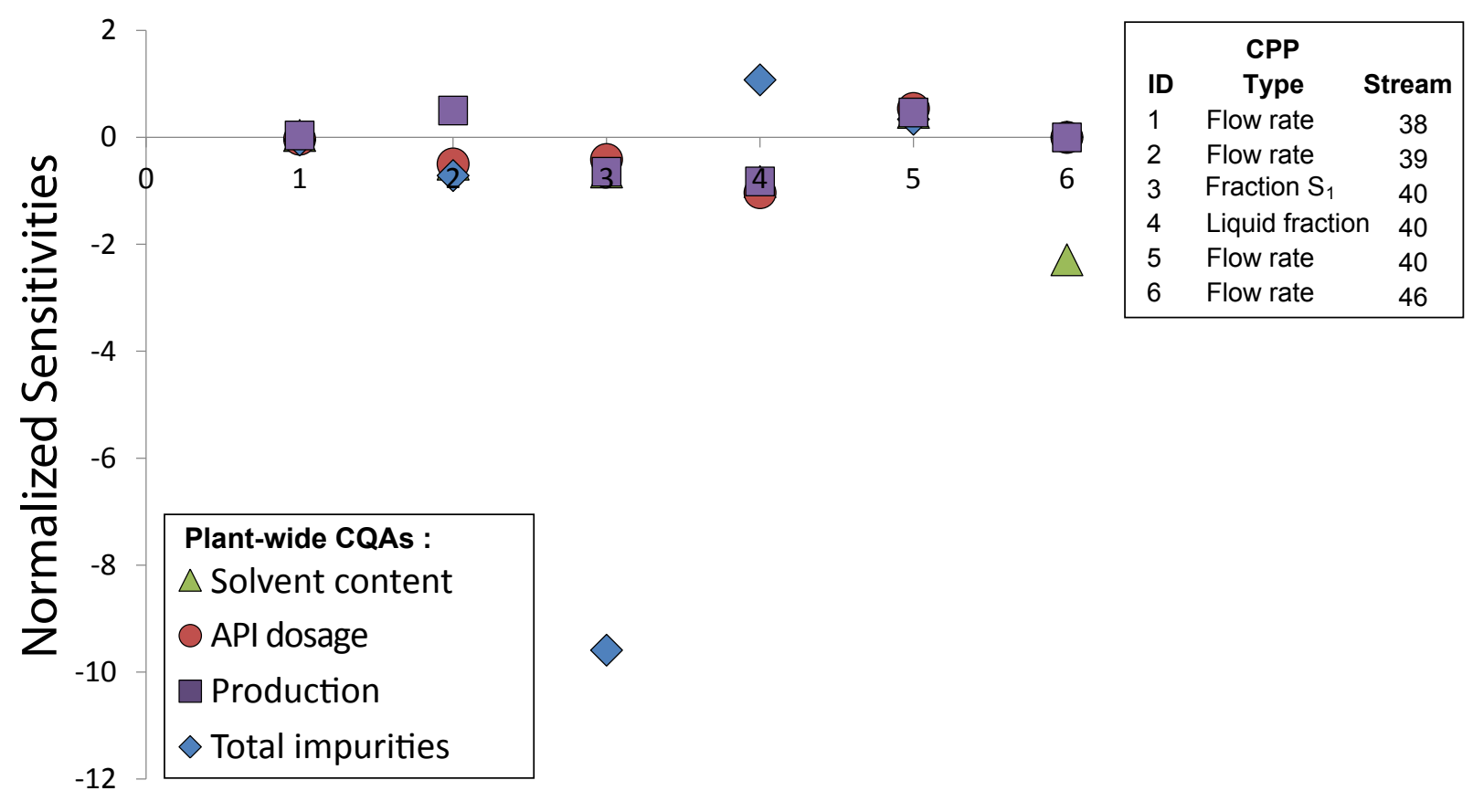

Figure 8: Steady-state values of the normalized sensitivities of the CQAs of the final product with respect to the available manipulated variables for Block $2 \mathrm{C}$ of the decomposition (see 5). In the legend, "Fraction $S_{1}$ " refers to the mass-based fraction of solvent $S_{1}$ in the liquid phase and "Liquid fraction" refers to the volume-based liquid fraction of the slurry. The CPPs with ID 4, 5, and 6 can be manipulated by changing the setpoints of control loops that are established around Block 2A (see 4). The numerical values of the sensitivities are available as Supplementary Material. 


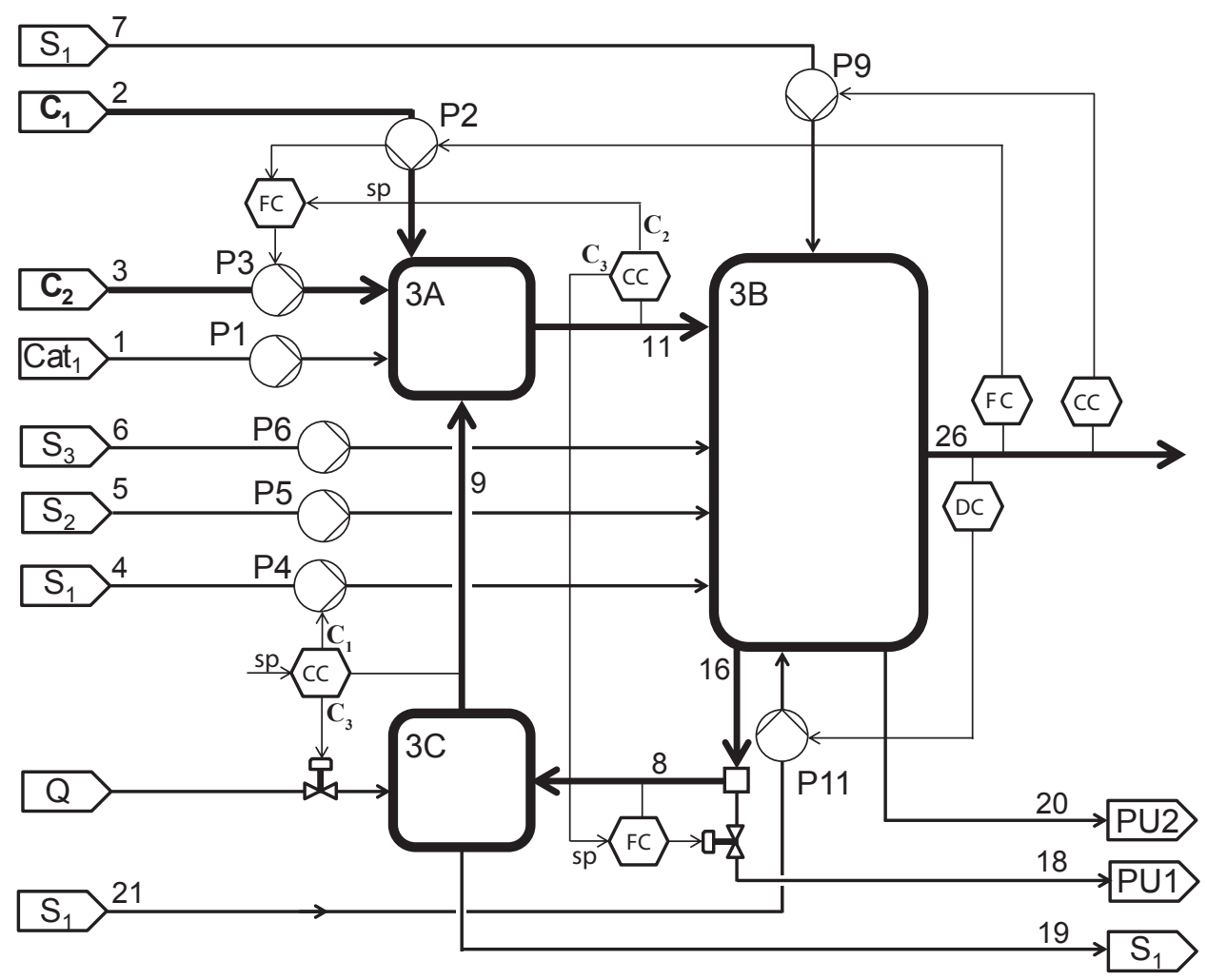

Figure 9: Representation of the continuous pharmaceutical pilot plant at Level 3 of the decomposition strategy. Block 2A from Level 2 (see 4) has been expanded to visualize the recycle structure. Block $3 \mathrm{~A}$ contains all unit operations related to the first reaction, which produces the intermediate impurity $\mathrm{I}_{1}$. Block $3 \mathrm{~B}$ represents all unit operations that are used to separate the reaction products into a stream that is rich in impurity $\mathrm{I}_{1}$ (Stream 26) and a stream that is rich in reactant $C_{1}$ (Stream 16), which is partially purged (Stream 18) and send to a flash evaporator (Block 3C) to remove solvent $S_{1}$ (Stream 19) before the material returns to the reaction section (Stream 9). The control loops aim to control the CQAs of the streams that constitute the recycle to minimize the propagation of disturbances to the CQAs of Stream 26, which are critical at a higher level of the decomposition. 


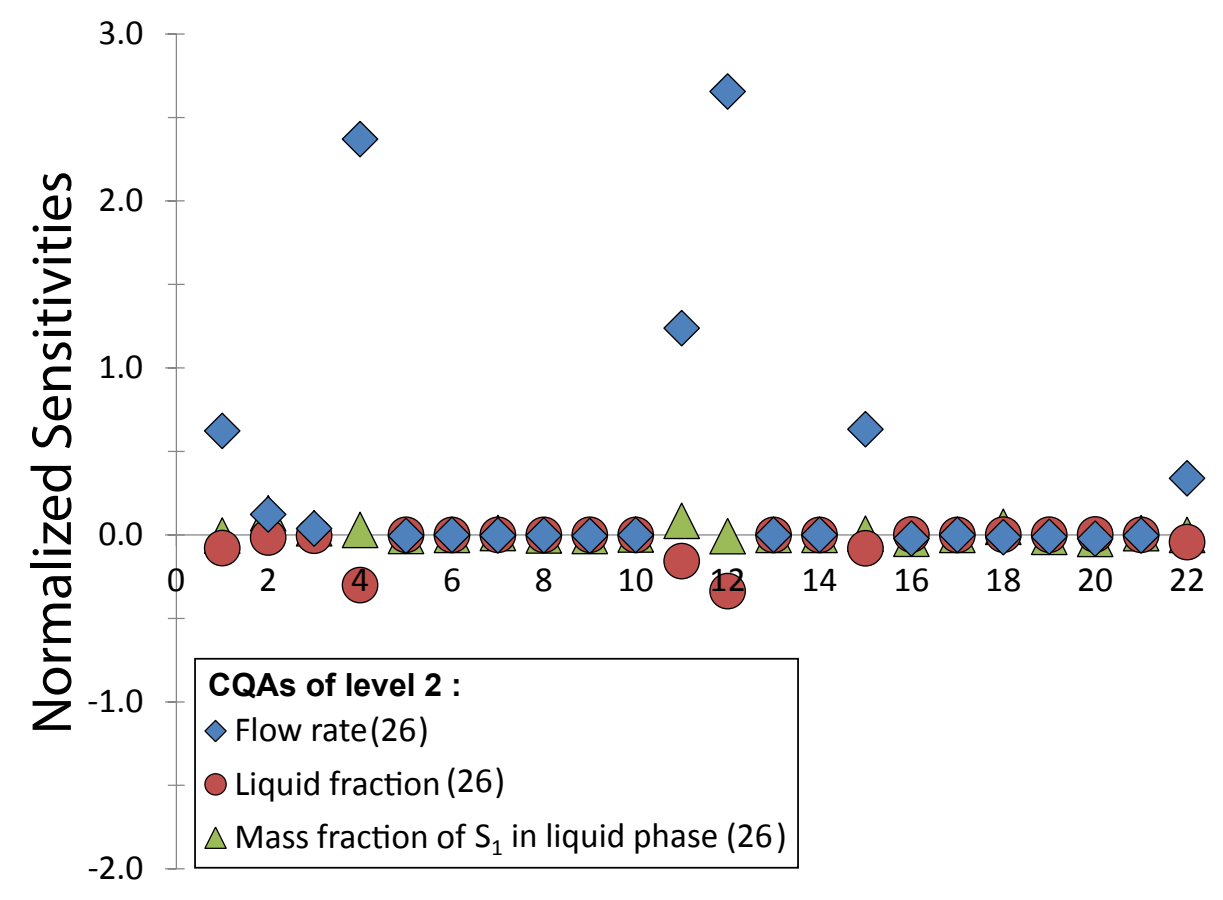

\begin{tabular}{|c|c|c|}
\hline & CPP & \\
\hline ID & Type & Stream \\
\hline 1 & Fraction $\mathrm{C}_{1}$ & 11 \\
\hline 2 & Fraction $\mathrm{C}_{2}$ & 11 \\
\hline 3 & Fraction Cat $_{1}$ & 11 \\
\hline 4 & Fraction $\mathrm{C}_{3}$ & 11 \\
\hline 5 & Fraction $\mathrm{I}_{2}$ & 11 \\
\hline 6 & Fraction $\mathrm{S}_{2}$ & 11 \\
\hline 7 & Fraction $\mathrm{S}_{1}$ & 11 \\
\hline 8 & Fraction $\mathrm{I}_{3}$ & 11 \\
\hline 9 & Fraction $\mathrm{I}_{1}$ & 11 \\
\hline 10 & Fraction $\mathrm{S}_{3}$ & 11 \\
\hline 11 & Flow rate & 11 \\
\hline 12 & Fraction $\mathrm{C}_{1}$ & 9 \\
\hline 13 & Fraction $\mathrm{C}_{2}$ & 9 \\
\hline 14 & Fraction Cat $_{1}$ & 9 \\
\hline 15 & Fraction $\mathrm{C}_{3}$ & 9 \\
\hline 16 & Fraction $\mathrm{I}_{2}$ & 9 \\
\hline 17 & Fraction $\mathrm{S}_{2}$ & 9 \\
\hline 18 & Fraction $\mathrm{S}_{1}$ & 9 \\
\hline 19 & Fraction $\mathrm{I}_{3}$ & 9 \\
\hline 20 & Fraction $\mathrm{I}_{1}$ & 9 \\
\hline 21 & Fraction $\mathrm{S}_{3}$ & 9 \\
\hline 22 & Flow rate & 9 \\
\hline
\end{tabular}

Figure 10: Steady-state values of the normalized sensitivities of the CQAs of Level 2 with respect to the CPPs at Level 3. The stream numbers refer to 1 . The fraction of the various compounds in the table with CPPs represents the mass-based fraction of a compound in the liquid phase. The liquid fractions are volume-based liquid fraction of a slurry. The numerical values of the sensitivities are available as Supplementary Material. 


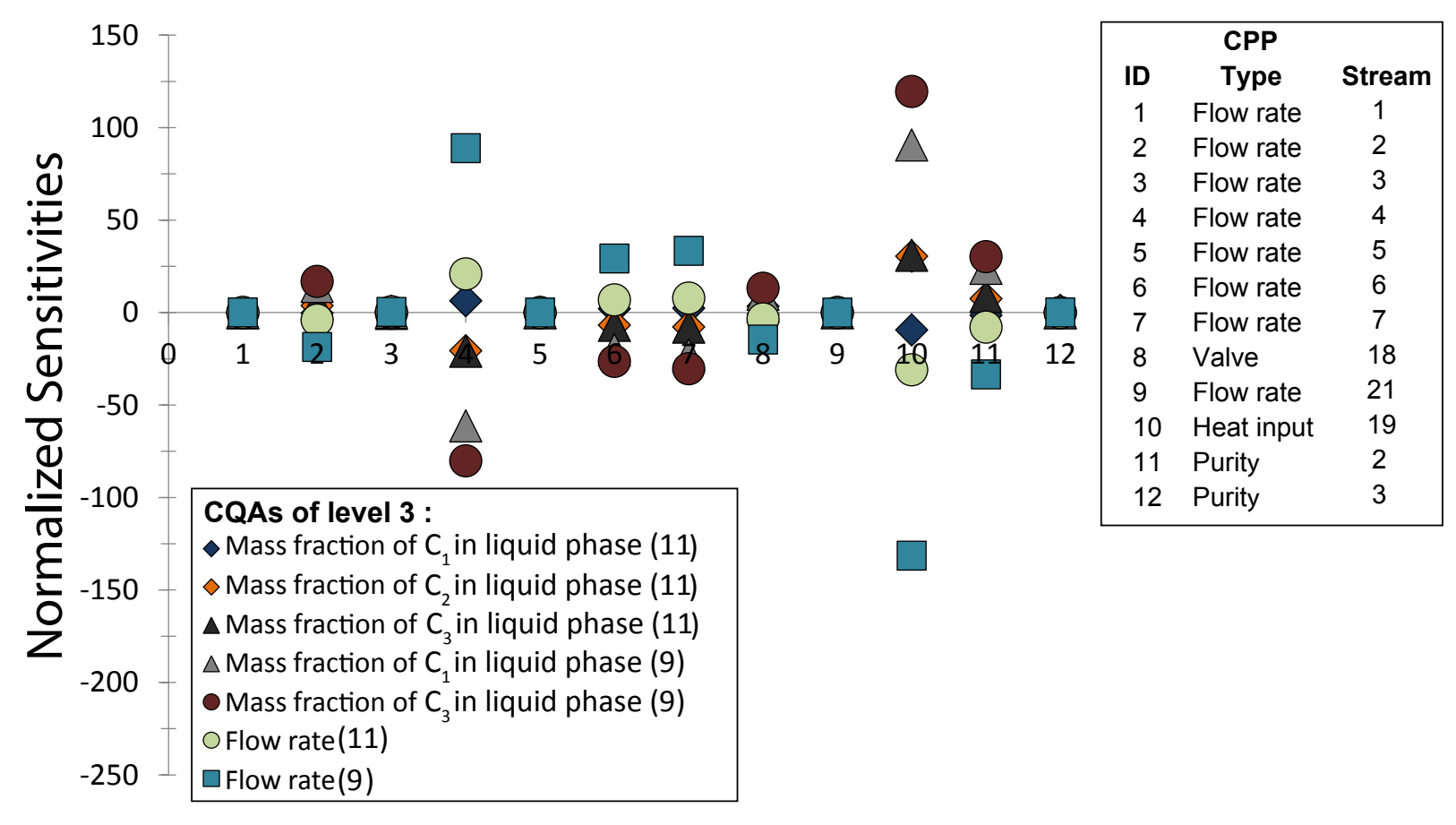

Figure 11: Steady-state values of the normalized sensitivities of CPPs of Streams 9 and 11 that have the largest impact on the CQAs of Stream 26 (see 10). The liquid fraction is the volume-based liquid fraction of the slurry. The numerical values of the sensitivities are available as Supplementary Material. 


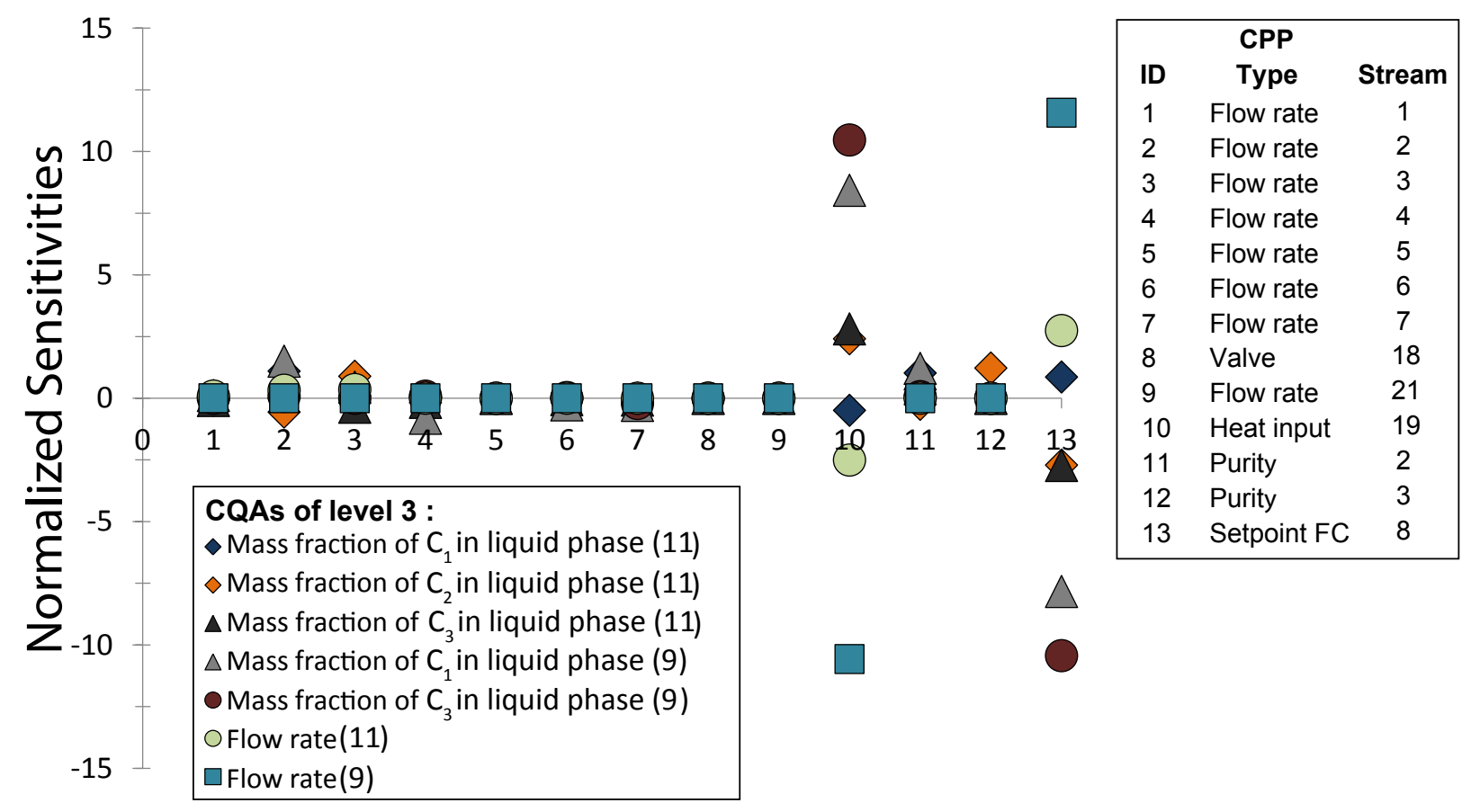

Figure 12: Steady-state values of the normalized sensitivities of CPPs of Streams 9 and 11 that have the largest impact on the CQAs of Stream 26 (see 10) with a controlled flow rate in the recycle. The liquid fraction is the volume-based liquid fraction of the slurry. The numerical values of the sensitivities are available as Supplementary Material. 


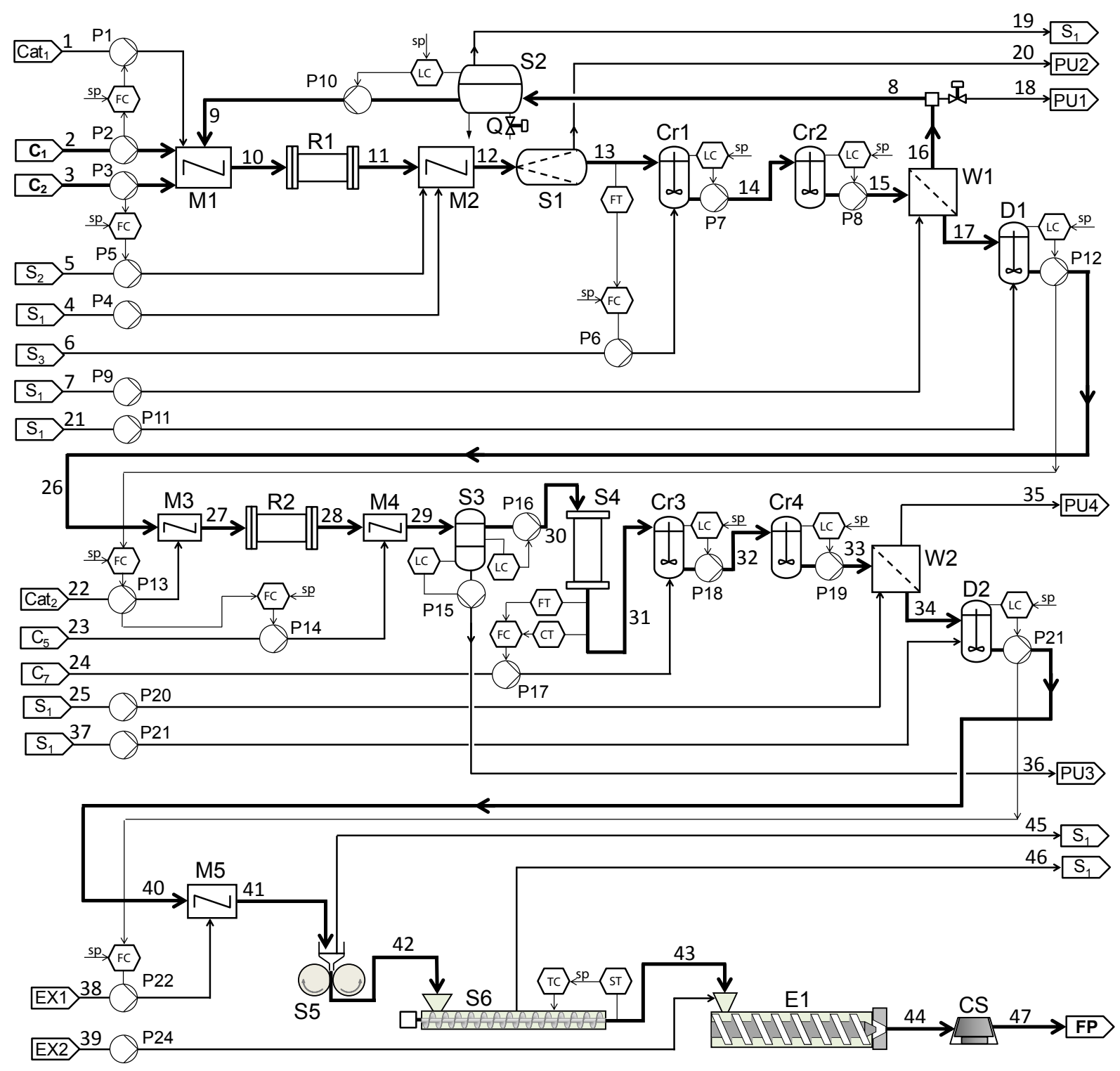

Figure 13: Representation of the continuous pharmaceutical pilot plant at the most detailed level of the decomposition strategy. The control structure contains all stabilizing control loops and the control loops that are synthesized at Level 4 of the decomposition to satisfy local control objectives. 


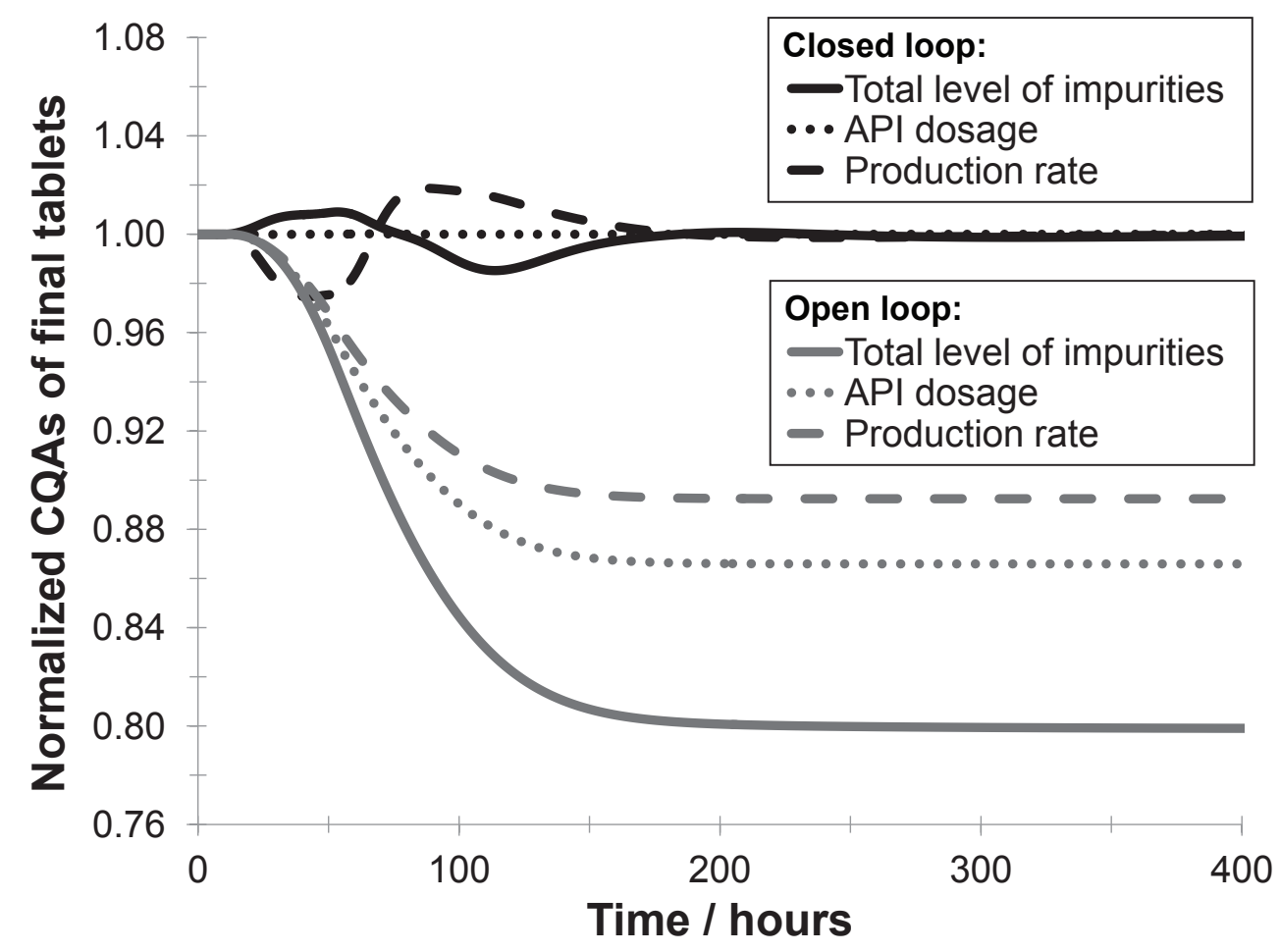

Figure 14: Dynamic development of three CQAs of the final product stream for a disturbance in the flow rate of fresh reactant $C_{1}$ (Case I). The black lines illustrate the closed-loop response and the gray lines illustrate the response with a stabilizing control layer only. The solid lines represent the dynamic development of the total level of impurities, the dotted lines represent the dynamic development of the API dosage, and the dashed lines represent the dynamic development of the production rate. 


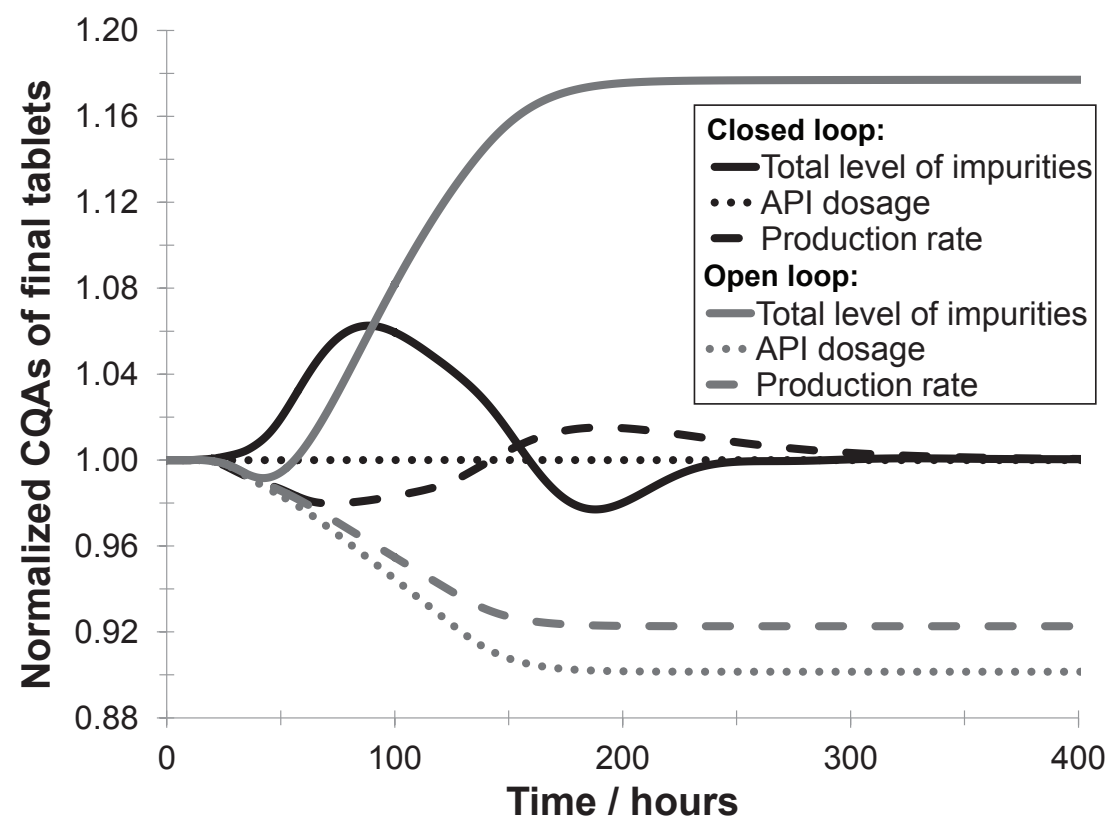

Figure 15: Dynamic development of three CQAs of the final product stream for a gradual change in the kinetics of the reactions in reactor 1 and 2 (Case II). The black lines illustrate the closedloop response and the gray lines illustrate the response with a stabilizing control layer only. The solid lines represent the dynamic development of the total level of impurities, the dotted lines represent the dynamic development of the API dosage, and the dashed lines represent the dynamic development of the production rate. 


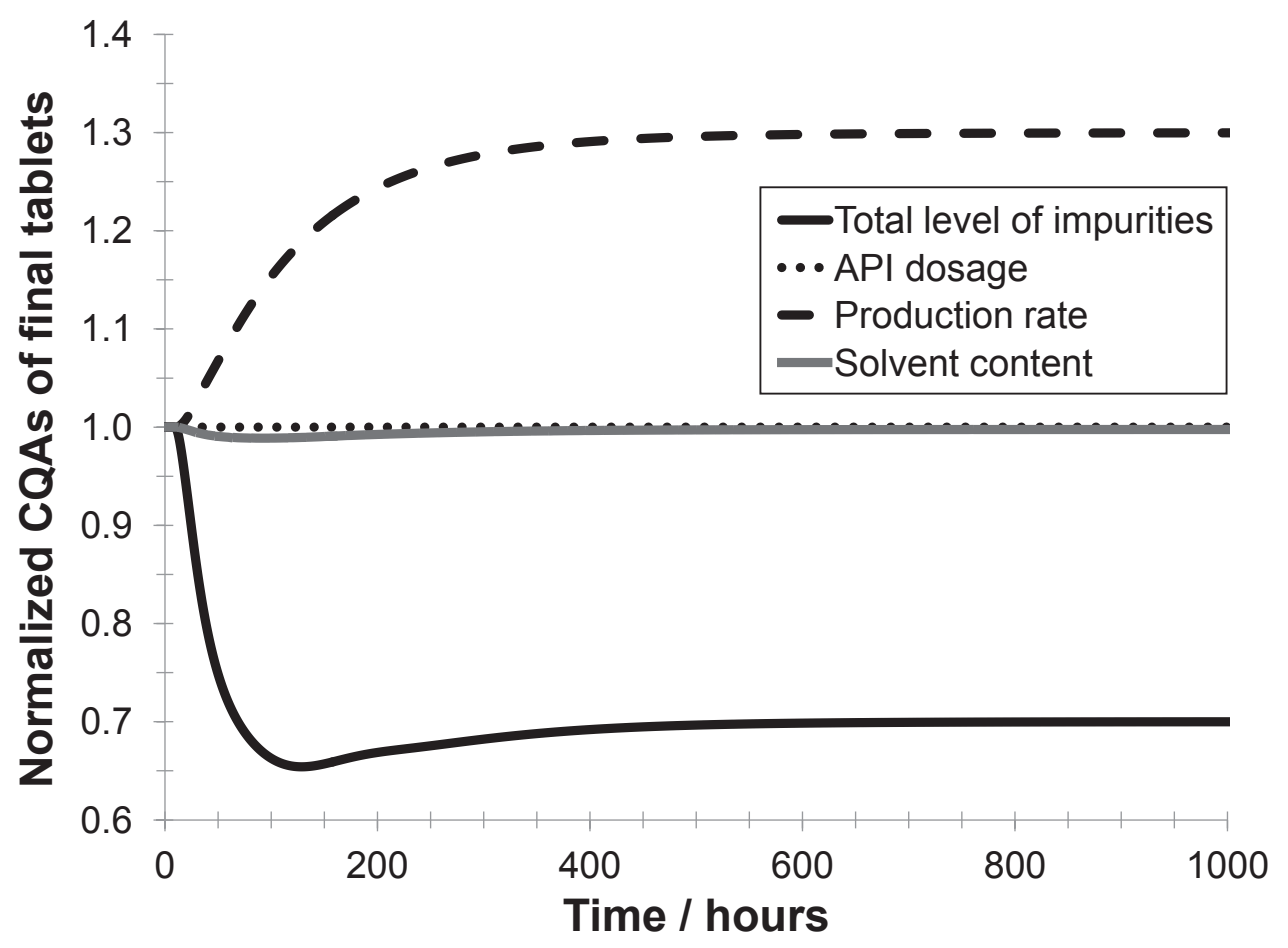

Figure 16: Dynamic development of four CQAs of the final product stream as a result of a stepchange in the setpoints of the production rate and total level of impurities at $t=0(+30 \%$ and $-30 \%$ respectively). The solid black (bottom) line represents the dynamic development of the total level of impurities, the dotted black line (circles) represents the dynamic development of the API dosage, the gray line represents the dynamic development of the solvent content, and the dashed black line represents the dynamic development of the production rate. 


\section{List of Tables}

1 Control loops of the stabilizing control layer. $V$ refers to volume and $F$ refers to flow rate. The abbreviations for unit operations and stream numbers are indicated in 1. . . . . . . . . . . . . . . . 49

2 Critical Quality Attributes of final tablets . . . . . . . . . . .

3 Tuning parameters of control loops. $F$ refers to flow rate $[\mathrm{kg} / \mathrm{h}], \varepsilon$ is a volumetric liquid fraction, $w$ is a weight fraction, $Q$ is a heat input [W], $T$ is a temperature [K], $S P$ refers to a setpoint, $V L$ refers to a control valve, and $V$ is a volume $\left[\mathrm{m}^{3}\right]$. The abbreviations of stream numbers and unit operations are indicated in $1 . \ldots \ldots . . . . . .$. 
Table 1: Control loops of the stabilizing control layer. $V$ refers to volume and $F$ refers to flow rate. The abbreviations for unit operations and stream numbers are indicated in 1 .

\begin{tabular}{cc}
$\begin{array}{c}\text { Controlled } \\
\text { variable }\end{array}$ & $\begin{array}{c}\text { Manipulated } \\
\text { variable }\end{array}$ \\
\hline$V_{C r 1}$ & $F_{14}$ \\
$V_{C r 2}$ & $F_{15}$ \\
$V_{D 1}$ & $F_{26}$ \\
$V_{S 3, O R}$ & $F_{30}$ \\
$V_{S 3, A Q}$ & $F_{36}$ \\
$V_{C r 3}$ & $F_{32}$ \\
$V_{C r 4}$ & $F_{33}$ \\
$V_{D 2}$ & $F_{40}$
\end{tabular}


Table 2: Critical Quality Attributes of final tablets

\begin{tabular}{lcc} 
Critical Quality Attribute & Allowable range & Unit \\
\hline Primary CQAs & & \\
Solvent Content & {$[0,0.0050]$} & $\mathrm{g} / \mathrm{g}$ \\
Total level of impurities & {$[0,0.008]$} & $\mathrm{g} / \mathrm{g}$ \\
Dosage of API & {$[0.42,0.48]$} & $\mathrm{g} / \mathrm{g}$ \\
\hline Secondary CQA & & \\
Production rate & {$[0.22,0.30]$} & $\mathrm{kg} / \mathrm{h}$
\end{tabular}


Table 3: Tuning parameters of control loops. $F$ refers to flow rate $[\mathrm{kg} / \mathrm{h}], \varepsilon$ is a volumetric liquid fraction, $w$ is a weight fraction, $Q$ is a heat input [W], $T$ is a temperature [K], $S P$ refers to a setpoint, $V L$ refers to a control valve, and $V$ is a volume $\left[\mathrm{m}^{3}\right]$. The abbreviations of stream numbers and unit operations are indicated in 1.

Variables

Nominal PI parameters

\begin{tabular}{cccccccc} 
Controlled $(\mathrm{CV})$ & Manipulated (MV) & Range MV & Setpoint CV & Level & $K_{c}$ & units & $\tau_{i}(\mathrm{~h})$ \\
\hline$\varepsilon_{26}$ & $F_{11}$ & {$[0,4.8]$} & $6.8 \mathrm{E}-01$ & 2 & $3.9 \mathrm{E}-02$ & $\mathrm{~m}^{3} / \mathrm{h}$ & 3.0 \\
$F_{26}$ & $F_{2}$ & $\geq 0$ & - & 2 & $3.8 \mathrm{E}-01$ & - & 30 \\
$w_{L, S_{1}, 26}$ & $F_{7}$ & {$[0,4.8]$} & $9.22 \mathrm{E}-01$ & 2 & $7.7 \mathrm{E}+00$ & $\mathrm{~kg} / \mathrm{h}$ & 3.0 \\
$\varepsilon_{40}$ & $F_{37}$ & {$[0,4.8]$} & $6.9 \mathrm{E}-01$ & 2 & $3.9 \mathrm{E}-02$ & $\mathrm{~m}^{3} / \mathrm{h}$ & 3.0 \\
$F_{40}$ & $S P_{F_{26}}$ & $\geq 0$ & - & 2 & $1.5 \mathrm{E}+00$ & - & 10 \\
$w_{L, S_{1}, 40}$ & $F_{25}$ & {$[0,4.8]$} & - & 2 & $7.7 \mathrm{E}+00$ & $\mathrm{~kg} / \mathrm{h}$ & 3.0 \\
$F_{47}$ & $S P_{F_{40}}$ & $\geq 0$ & $2.6 \mathrm{E}-1$ & 2 & $2.6 \mathrm{E}-02$ & - & 3 \\
$w_{I M P, 47}$ & $S P_{w_{L, S}, 40}$ & {$[0.85,0.98]$} & $6.0 \mathrm{E}-03$ & 2 & $-1.6 \mathrm{E}+00$ & - & 13 \\
$w_{A P I, 47}$ & $F_{39}$ & {$[0,4.8]$} & $4.5 \mathrm{E}-01$ & 2 & $-2.5 \mathrm{E}-01$ & $\mathrm{~kg} / \mathrm{h}$ & 0.050 \\
$w_{C_{2}, 11}$ & $S P_{F_{3}}$ & $\geq 0$ & $2.8 \mathrm{E}-01$ & 3 & $2.5 \mathrm{E}+00$ & - & 2.0 \\
$w_{C_{3}, 11}$ & $S P_{F_{8}}$ & $\geq 0$ & $3.9 \mathrm{E}-01$ & 3 & $-8.7 \mathrm{E}-01$ & $\mathrm{~kg} / \mathrm{h}$ & 12.0 \\
$F_{8}$ & $V L_{18}$ & {$[0,1]$} & - & 3 & $6.7 \mathrm{E}-01$ & $\mathrm{~h} / \mathrm{kg}$ & 0.10 \\
$w_{C_{1}, 9}$ & $F_{4}$ & {$[0.4,4]$.} & $3.9 \mathrm{E}-01$ & 3 & $-1.1 \mathrm{E}+00$ & $\mathrm{~kg} / \mathrm{h}$ & 15 \\
$w_{C 3}, 9$ & $Q$ & {$[0,556]$} & $1.1 \mathrm{E}-01$ & 3 & $7.4 \mathrm{E}+01$ & $\mathrm{~W}$ & 2.0 \\
$V_{C r 1}$ & $F_{14}$ & {$[0,4.8]$} & $8.0 \mathrm{E}-3$ & 4 & $2.4 \mathrm{E}+03$ & $\mathrm{~kg} / \mathrm{m}^{3} \mathrm{~h}$ & - \\
$V_{C r 2}$ & $F_{15}$ & {$[0,4.8]$} & $8.0 \mathrm{E}-3$ & 4 & $2.4 \mathrm{E}+03$ & $\mathrm{~kg} / \mathrm{m}^{3} \mathrm{~h}$ & - \\
$V_{S 2}$ & $F_{9}$ & {$[0,4.8]$} & $3.0 \mathrm{E}-4$ & 4 & $4.8 \mathrm{E}+03$ & $\mathrm{~kg} / \mathrm{m}^{3} \mathrm{~h}$ & - \\
$V_{D 1}$ & $F_{26}$ & {$[0,4.8]$} & $3.0 \mathrm{E}-3$ & 4 & $4.8 \mathrm{E}+03$ & $\mathrm{~kg} / \mathrm{m}^{3} \mathrm{~h}$ & - \\
$V_{S 3, A Q}$ & $F_{36}$ & {$[0,4.8]$} & $1.0 \mathrm{E}-3$ & 4 & $9.6 \mathrm{E}+04$ & $\mathrm{~kg} / \mathrm{m}^{3} \mathrm{~h}$ & - \\
$V_{S 3, O R}$ & $F_{30}$ & {$[0,4.8]$} & $2.0 \mathrm{E}-3$ & 4 & $9.6 \mathrm{E}+03$ & $\mathrm{~kg} / \mathrm{m}^{3} \mathrm{~h}$ & - \\
$V_{C r 3}$ & $F_{32}$ & {$[0,4.8]$} & $8.0 \mathrm{E}-3$ & 4 & $2.4 \mathrm{E}+03$ & $\mathrm{~kg} / \mathrm{m}^{3} \mathrm{~h}$ & - \\
$V_{C r 4}$ & $F_{33}$ & {$[0,4.8]$} & $8.0 \mathrm{E}-3$ & 4 & $2.4 \mathrm{E}+03$ & $\mathrm{~kg} / \mathrm{m}^{3} \mathrm{~h}$ & - \\
$V_{D 2}$ & $F_{40}$ & {$[0,4.8]$} & $3.0 \mathrm{E}-3$ & 4 & $4.8 \mathrm{E}+03$ & $\mathrm{~kg} / \mathrm{m}^{3} \mathrm{~h}$ & - \\
$w_{S_{1}, 43}$ & $T_{S 5}$ & {$[313,363]$} & $2.0 \mathrm{E}-03$ & 4 & $1.7 \mathrm{E}+06$ & $\mathrm{~K}$ & -
\end{tabular}

